Open Access

\title{
Occlusal rehabilitation in patients with congenitally missing teeth-dental implants, conventional prosthetics, tooth autotransplants, and preservation of deciduous teeth-a systematic review
}

Hendrik Terheyden ${ }^{1 *}$ and Falk Wüsthoff ${ }^{2}$

\begin{abstract}
Background: Implant patients with congenitally missing teeth share some common charateristics and deserve special attention.

Methods: The PICO question was "In patients with congenitally missing teeth, does an early occlusal rehabilitation with dental implants in comparison to tooth autotransplants, conventional prosthetics on teeth or preservation of deciduous teeth have better general outcomes in terms of survival, success and better patient centered outcomes in terms of quality of life, self-esteem, satisfaction, chewing function?"

After electronic database search, a total of 63 relevant studies were eligible, of which 42 qualified for numerical data synthesis, 26 being retrospective studies. A data synthesis was performed by weighted means for survival/success/ annual failure rates.

Results: The mean survival of implants was $95.3 \%$ (prosthesis survival $97.8 \%$ ), autotransplants $94.4 \%$, deciduous teeth $89.6 \%$, and conventional prostheses $60.2 \%$. The implant survival in children, adolescents, and adults was $72.4,93.0$, and $97.4 \%$. Annual failure rates of implants $3.317 \%$, autotransplants $1.061 \%$, deciduous teeth $0.908 \%$, and conventional prostheses $5.144 \%$ indicated better results for natural teeth and more maintenance needs for the both prosthetic treatments. The mean OHIP score was 27.8 at baseline and a mean improvement of 14.9 score points was reported after implant prosthetics. The mean satisfaction rates were 93.4 (implants), 76.6 (conventional prostheses), 72.0 (autotransplants), and $65.5 \%$ (orthodontic space closure).

Conclusions: In synopsis of general and patient-centered outcomes, implants yielded the best results, however, not in children $<13$ years. Autotransplants and deciduous teeth had low annual failure rates and are appropriate treatments in children and adolescents at low costs. Conventional prosthetics had lower survival/ success rates than the other options. Due to heterogeneity and low number of studies, patient-reported outcomes in this review have to be interpreted with caution.
\end{abstract}

Keywords: Hypodontia; Oligodontia; Anodontia; Tooth aplasia; Tooth agenesis; Congenitally missing teeth; Tooth autotransplantation; Deciduous tooth; Dental implants; Ectodermal dysplasia

\footnotetext{
* Correspondence: Terheyden@rkh-kassel.de

'Department of Oral and Maxillofacial Surgery, Red Cross Hospital, Hansteinstr. 29, D-34121 Kassel, Germany

Full list of author information is available at the end of the article
} 


\section{Background}

Congenitally missing teeth, also called hypodontia, is the most frequent human malformation. The prevalence of hypodontia in white populations is estimated to be $5.5 \%$, with a higher incidence in women than in men. Hypodontia varies in severity, from a single missing tooth to the absence of all permanent teeth called anodontia. Oligodontia is usually defined as the absence of 6 or more permanent teeth, the third molars excluded, and its prevalence is estimated at $0.14 \%$ in the white population [1]. Hypodontia can occur in isolation (non-syndromal) or as a part of numerous inherited syndromes of which the different forms of ectodermal dysplasia are the predominant entity [2]. Patients with congenitally missing teeth comprise a special group of patients deserving special attention, especially when dental implants are needed.

In cases with congenitally missing teeth, the defect in the dentition occurs very early in life, in contrast to many other implant patients who lost their teeth due to caries or periodontitis at later stages. The early time point has an advantage that the young patients are usually well adapted to the defects. However, prosthetic treatments are often necessary already in childhood. In childhood and adolescence, prosthetic treatments can be complicated, because teeth should not be ground as abutments for crowns due to the large pulp cavity, and dentures may not be splinted if the jaws still grow. It is also questionable whether dental implants can be placed before termination of growth due to the well-known problems of secondary infraocclusion due to the ankylotic healing of osseointegrated implants and due to other biological reasons [3]. Furthermore, children and their young parents and families often have a cost problem, since unlike other implant patient groups, the tooth defects appear in early phases of life when the income is low or needed elsewhere. In some public health systems, occlusal rehabilitation in childhood and adolescence is covered by public insurances and has to be finished before the 18th year of life.

The local implant site can be special, too. A site with aplasia of the primary or secondary tooth usually is different from implant sites in conventional implant patients. Usually, there is a severe lack of alveolar bone width and often height and bone has never been there, since alveolar bone is unlike the basal jaw bone, a development of the erupting tooth. In addition, bone quality can be more cortical and brittle than in conventional implant sites, which can influence implant placement as well as orthodontic tooth movement. The same applies to the fixed masticatory gingiva in a site with tooth aplasia, which can be narrow or missing at all. Due to these common general properties of an implant site with tooth agenesis irrespective of the number of missing teeth, this systematic review includes articles with single or few missing teeth (mild hypodontia), multiple $(>6)$ missing teeth (oligodontia), and also total absence of teeth (anodontia).

There may be mainly four or five treatment options for occlusal rehabilitation in cases with congenitally missing teeth, including dental implant-borne prosthetics. First option is the preservation of a primary deciduous tooth. Such decision has to be made first, if a primary tooth is still present in the site due to missing eruption of a permanent successor. A second option may be the autotransplantation of other teeth, if such transplants are available. This is a well-accepted method in childhood, since tooth autotransplants can heal with a functional periodontium which enables orthodontic movement and enables the tooth to participate in growth of the alveolar crest. Furthermore, tooth transplants in childhood have a better prognosis, when root development is still incomplete and the apical foramen is still open, compared to mature teeth in adults with closed foramen. A third option is conventional prosthetics on teeth, which in childhood will typically include unsplinted overdentures, which should not interfere with jaw growth or resin-bonded bridges, since juvenile teeth should not be ground for crowns. The fourth option is dental implants. Each of the four options has its advantages and limitations and a differential indication has to be made in every single case of congenitally missing teeth. The fifth option may be orthodontic space closure as a single treatment. Of course, adjunctive orthodontic treatment is a very important part of occlusal rehabilitation in patients with congenitally missing teeth. However, this is not in the focus of this systematic review, because this therapy is not generally applicable to cases with multiple missing teeth.

To the knowledge of the authors, narrative literature reviews [4-7] and consensus meetings [8-10] have been published on the topic in the literature. There is only one systematic review without numerical meta-analysis [11]. In the latter, Yap and Klineberg addressed studies on dental implants in patients with ectodermal dysplasia (ED) and tooth agenesis but not on the alternative treatments. They found documented implant survival rates of 88.5$97.6 \%$ in ED cases and $90-100 \%$ in tooth agenesis. The authors concluded that implants placed in adolescent patients with ED do not have a significant negative influence on facial growth and that implants in ED patients younger than 18 years have a high risk of failure.

The aim of this study is a systematic review of the literature of treatment of patients with congenitally missing teeth and a meta-analysis in from of weighted means of survival and success data. The aim is to elucidate the role of dental implants in the group of patients with congenitally missing teeth in comparison with the other treatment options. In addition to the general treatment 
outcome parameters, the aim was also to include patient-centered and patient-reported outcome parameters. This paper was prepared as the basis of a consensus meeting of the German Implant Association to be held on 9th-10th of September 2015 in Aerzen, Germany.

\section{Methods}

This systematic review was structured and performed according to the preferred reporting items of the PRISMA statement [12].

\section{Focused question}

The focused question serving for literature search was structured according to the PICO format (Table 1) "In patients with congenitally missing teeth, does an early occlusal rehabilitation with dental implants in comparison to tooth autotransplants, conventional prosthetics on teeth or preservation of deciduous teeth have better general outcomes in terms of survival, success and better patient centered outcomes in terms of quality of life, self-esteem, satisfaction, chewing function?".

\section{Search strategy}

PubMed of the US National Library of Medicine and EMBASE were used as electronic databases to perform a systematic search for relevant articles published in the dental literature between 1980 up to 31 May 2015.

A first probatory screening using only the MeSH terms "anodontia" and "dental implants" yielded too few results. It became clear that there are numerous synonyms of anodontia, which had to be included in the search (Table 1). A search strategy based on the elements of the PICO question was constructed: (tooth OR teeth OR dental) AND ((anodontia OR aplas* OR agenesis OR oligodontia OR hypodontia OR developmentally absent OR congenitally missing OR noncarious OR birth defect OR ectodermal dysplasia) AND ((rehabilitation OR dental implant* OR bone augment* OR alveolar bone grafting)

Table 1 Logical deduction of the literature search phrase from the PICO questionable

\begin{tabular}{|c|c|c|c|}
\hline Patients & Intervention & Control & Outcomes \\
\hline \multirow{9}{*}{$\begin{array}{l}\text { Patients with congenitally missing } \\
\text { teeth }\end{array}$} & Rehabilitation & Tooth autotransplants & General: \\
\hline & Dental implants & Preservation of deciduous teeth & Implant/tooth survival/success \\
\hline & Bone augmentation & $\begin{array}{l}\text { Conventional prosthodontic } \\
\text { treatment }\end{array}$ & Prosthesis survival/success \\
\hline & & Orthodontic treatment & Craniofacial growth \\
\hline & & & Patient reported: \\
\hline & & & Quality of life \\
\hline & & & Self-esteem \\
\hline & & & Satisfaction \\
\hline & & & Chewing function \\
\hline \multicolumn{4}{|l|}{$\begin{array}{l}\text { Synonyms, search terms, search } \\
\text { phrase }\end{array}$} \\
\hline - Anodontia (MeSH) & - Rehabilitation & - Autotransplan* & - Survival \\
\hline - Hypodontia & - Dental implan* (MeSH) & - Crown (MeSH) & - Success \\
\hline - Oligodontia & - Alveolar bone grafting (MeSH) & - Dentur* (MeSH) & - Growth \\
\hline • Tooth aplasia & - Bone augmentation & - Dental Prosthe* (MeSH) & - Quality of life (MeSH) \\
\hline - Tooth agenesis & & - Orthodonti* (MeSH) & - Satisfaction \\
\hline - Congenitally missing teeth & & • Tooth deciduous (MeSH) & - Self-esteem \\
\hline - Developmentally absent teeth & & & - Chewing \\
\hline - Birth defects & & & - Masticat* (MeSH) \\
\hline - Noncarious defects & & & - Masticat* (MeSH) \\
\hline \multicolumn{4}{|l|}{ - Ectodermal dysplasia } \\
\hline AND (tooth OR teeth OR dental) & & & \\
\hline
\end{tabular}

Boolean operators: (Within column OR, columns AND except intervention and control OR) AND (tooth OR teeth OR dental) NOT cancer, ophthalm*, brain, cataract

Filter: humans

Search phrase

(tooth OR teeth OR dental) AND ((anodontia OR aplas* OR agenesis OR oligodontia OR hypodontia OR developmentally absent OR congenitally missing OR noncarious OR birth defect OR ectodermal dysplasia) AND ((rehabilitation OR dental implant* OR bone augment* OR alveolar bone grafting) OR (autotransplan* OR crown OR denture* OR dental prosth* OR orthodont* OR deciduous )) AND (survival OR success OR growth OR quality of life OR satisfaction OR self-esteem OR chewing OR masticat*) NOT cancer NOT ophthalm* NOT brain NOT cataract)) 
OR (autotransplan* OR crown OR denture* OR dental prosth* OR orthodont* OR deciduous )) AND (survival OR success OR growth OR quality of life OR satisfaction OR self-esteem OR chewing OR masticat*) NOT cancer NOT ophthalm* NOT brain NOT cataract)).

Screening was performed independently by the two authors. Disagreement regarding inclusion during the first and second stage of study selection was resolved by discussion.

Electronic search was complemented by an iterative hand-search in the reference lists of the already identified articles. If required, the corresponding authors were contacted and requested to provide missing data or information by email.

\section{Study inclusion and exclusion criteria}

During the first stage of study selection, the titles and abstracts were screened and evaluated according to the following inclusion criteria:

(1)English language.

(2) Retrospective and prospective clinical trials, observational studies, cross sectional studies, cohort studies, case series.

During this procedure, the pre-selected publications were evaluated according to the following exclusion criteria:

(1)Inclusion of minimum 5 patients (exclusion of case reports).

(2)Inadequate case definition or missing follow-up times.

(3)Double publication of the same sample

(4) Lack of clinical data

(5)Studies in cleft lip and palate patients

\section{Quality and risk of bias assessment of selected studies}

A quality assessment of all selected full-text articles was performed. It made no sense to use the Cochrane collaborations' tool for assessing risk of bias for randomized controlled studies since the majority of the included studies were retrospective case series. Instead, a system modified from the US Agency for Healthcare Research and Quality Methods Guide for Comparative Effectiveness Reviews was used, which asked for the sources of possible bias [13]. The criteria were each judged with low, medium, and high risk of bias: case selection bias and confounding, attrition bias (loss of participants), detection bias (reliable measures?), reporting bias (selective or incomplete reporting), followed by a summary of the risk. Exclusion and quality assessment was performed independently by both authors. Disagreements were resolved by discussion.

\section{Data extraction}

A data extraction template was generated and based on the treatment types for the general outcome parameters and for the patient-centered outcome parameters. Due to incomplete reporting, old studies, and changing definitions in some papers, the required data on survival and success were often not found directly listed in the publications. In this case, they had to be retrieved from side informations or recalculated from tables. The following rules were applied: survival meant that the unit (implant, tooth, prosthesis) was reported to be present in the oral cavity. Success definition of an implant followed the criteria of Buser and coworkers [14] which are the following: absence of persistent subjective complaints such as pain, foreign body sensation and/or dysesthesia, absence of a peri-implant infection with suppuration, absence of mobility, absence of a continuous radiolucency (severe bone resorption) around the implant. A prosthesis either conventional or implant borne was counted as a success, if there were no complications reported like fracture, soft tissue recessions, or documented treatment needs. A deciduous tooth was counted as a success, if there was no ankylosis and infraocclusion reported. A tooth autotransplant was regarded as a success if there were no reports of ankylosis or severe root resorption, infection, or mobility.

\section{Statistics and data synthesis}

For data synthesis, the survival and success data of the individual studies were pooled by the weighted mean method and $95 \%$ confidence intervals were calculated as estimations of variance. A meta-analysis was not possible due to the structure of the underlying survival success data as simple percentages without a measure of variance and without control groups in most studies. The survival/ success data were weighted both based on patients and units (either implants/teeth/prostheses). Because the studies showed large differences in follow-up times, these were standardized by calculating annual failure rates by dividing the success and survival data through the follow-up time in years. All spreadsheet calculations and statistics were made with the Microsoft Excel program.

\section{Results}

\section{Study selection}

A total of 1508 potentially relevant titles and abstracts were found by the electronic search. Twelve titles found additionally by evaluation of reference lists of included articles were added. During first screening, 1020 publications were excluded based on database information. One hundred twenty-eight full-text articles were thoroughly evaluated. A total of 65 papers had to be excluded at this stage because they did not fulfill the inclusion criteria of 
the present systematic review. Sixty-three articles went into qualitative assessment (Fig. 1). One article had to be excluded or pooled with another because of possible double publication of the same cohort (Grecchi (b) [15]), one article because of missing follow-up times (Hvaring [16]), one because of missing clinical data (Kjaer [17]), and two articles because of missing numerical data (Dellavia [18, 19]). The study of Bergendal [20] could be kept in the analysis after contacting the author for clarification (no follow-up time reported). The implants in that study were observed only over the healing period (estimated 6 months in average). Because of too few studies and incompletely reported data studies on orthodontic gap closure, facial growth and masticatory performance were not included into the quantitative data synthesis. Forty-two studies were included in the assessment of general outcome parameter survival and success. For 16 studies on patient-centered variables, also weighted means were calculated.

\section{Evaluation of study quality and risk of bias}

The majority $(n=25)$ of the 42 studies were retrospective, 14 were prospective including one RCT 2 were cross sectional studies, and 1 remained unclear. Despite a low evidence level in terms of study design, there were no major concerns about risk of bias. The 30/42 studies were rated with a low risk of bias, and 12/42 had a medium risk. No study had a high risk of bias and consequently no further study was excluded at this stage because of bias (Table 2).

\section{Studies on general outcome parameters \\ Studies on dental implants and implant-supported prosthetics}

A total of 19 studies with a mean follow-up time of 4.6 years (maximum 15.1 years) was included (Table 3 ). Most studies were of retrospective character. The heterogeneity of the studies concerning survival and success data was low and acceptable for the numerical data synthesis. The studies showed some heterogeneity in patient inclusion, because in some studies, missing single teeth and treatments with single crowns on implants were mixed with severe oligodontia and varying prosthetic treatment including overdentures on implants. Also, management of bone defects and necessity of bone grafting were heterogenous between the studies. This heterogeneity did not lead to exclusion from the present numerical evaluation. The study of Bergendal and coworkers [20] was considered important, because a high ratio of implant failures was reported in childhood. It was one of the very few studies in the literature on implantation in childhood. However, no follow-up time was reported because it was a crosssectional survey. The author was contacted via email

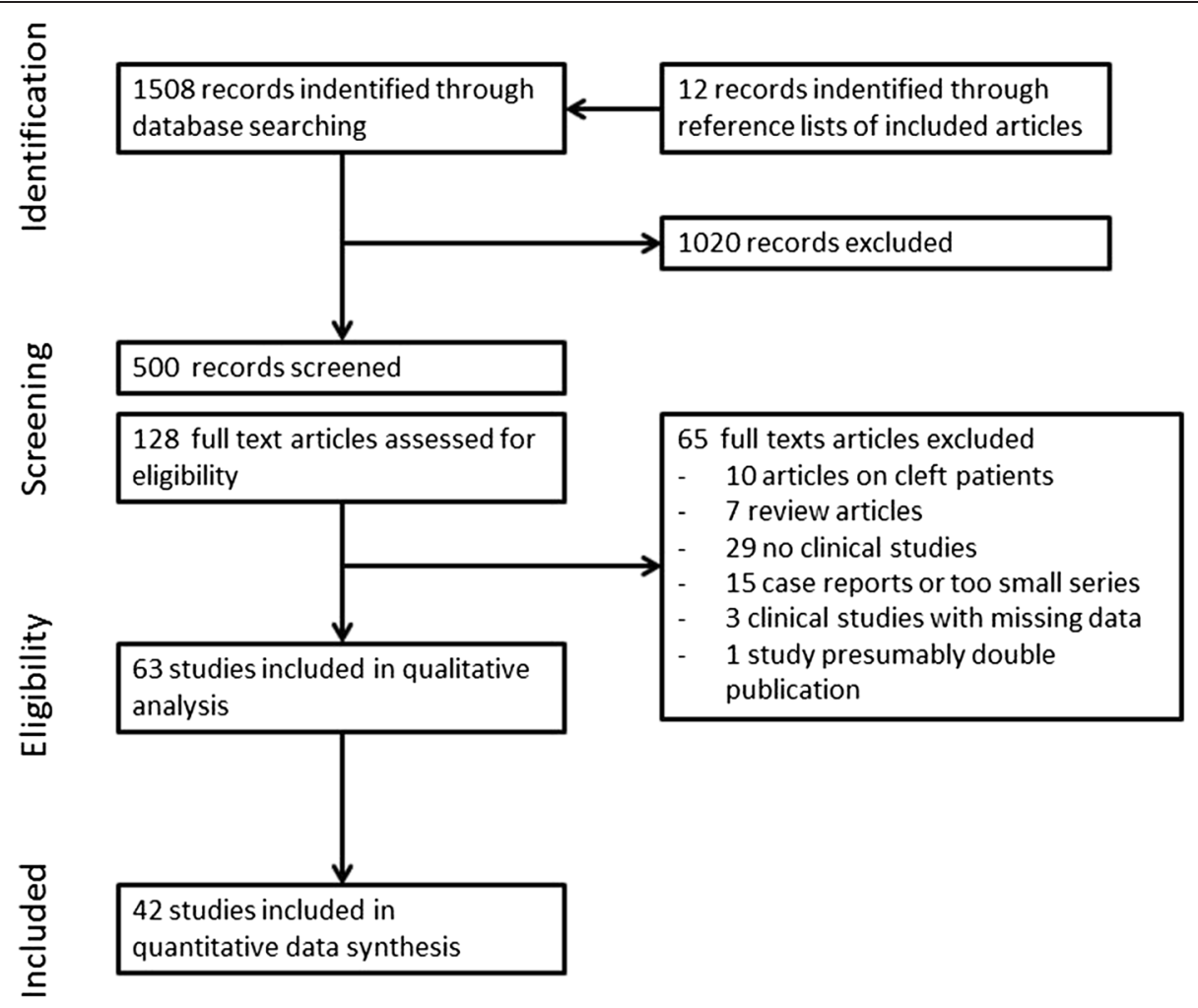

Fig. 1 Flow diagram of literature search and inclusion 
Table 2 Summary of the selected studies and quality assessement

\begin{tabular}{|c|c|c|c|c|c|c|c|c|}
\hline Author & Year & Study type & $\begin{array}{l}\text { Case selection } \\
\text { bias (homogeneity } \\
\text { and confounders) }\end{array}$ & $\begin{array}{l}\text { Performance } \\
\text { bias (fidelity } \\
\text { to protocol) }\end{array}$ & $\begin{array}{l}\text { Attrition } \\
\text { bias (loss } \\
\text { of participants) }\end{array}$ & $\begin{array}{l}\text { Detection } \\
\text { bias (reliable } \\
\text { measures) }\end{array}$ & $\begin{array}{l}\text { Reporting } \\
\text { bias (selective } \\
\text { reporting or } \\
\text { conflicting interests) }\end{array}$ & $\begin{array}{l}\text { Summary } \\
\text { assessments } \\
\text { Risk of bias }\end{array}$ \\
\hline \multicolumn{9}{|l|}{ Implant studies } \\
\hline Ledermann [40] & 1993 & Retrospect & M & M & $L$ & M & $\mathrm{H}$ & M \\
\hline Kearns [41] & 1999 & ProspObs & L & L & L & $\mathrm{L}$ & M & $\mathrm{L}$ \\
\hline Thilander [3] & 2001 & ProspObs & L & L & L & L & L & L \\
\hline Guckes [42] & 2002 & ProspObs & M & L & $L$ & $\mathrm{~L}$ & M & $\mathrm{L}$ \\
\hline Sweeney [43] & 2005 & Retrospect & $\mathrm{L}$ & L & L & $\mathrm{L}$ & M & L \\
\hline Finnema [27] & 2005 & Retrospect & $\mathrm{L}$ & L & M & $L$ & M & $\mathrm{L}$ \\
\hline Poggio [44] & 2005 & Retrospect & M & M & M & M & M & M \\
\hline Zarone [45] & 2006 & ProspObs & $\mathrm{L}$ & L & $\mathrm{L}$ & L & L & L \\
\hline Becelli [46] & 2007 & Retrospect & $\mathrm{L}$ & L & L & $L$ & M & $L$ \\
\hline Bergendal [20] & 2008 & Survey & M & L & $\mathrm{H}$ & M & M & M \\
\hline Dueled [26] & 2008 & Retrospect & M & L & M & $L$ & L & $\mathrm{L}$ \\
\hline Krieger [25] & 2009 & Retrospect & M & L & L & M & M & $\mathrm{L}$ \\
\hline Degidi [47] & 2009 & RCT & L & $L$ & L & L & L & L \\
\hline Creton [1] & 2010 & Retrospect & L & L & M & $\mathrm{L}$ & M & $\mathrm{L}$ \\
\hline Grecchi [15](a), [22](b) & 2010 & Retrospect & $\mathrm{H}$ & M & M & L & L & M \\
\hline Nissan [48] & 2011 & Unclear & $\mathrm{H}$ & L & M & M & M & M \\
\hline Heuberer [38] & 2012 & Retrospect & L & $L$ & L & $\mathrm{L}$ & M & L \\
\hline Hosseini [49] & 2013 & ProspObs & L & $L$ & L & L & L & $L$ \\
\hline Zou [50] & 2014 & Retrospect & M & $L$ & L & L & $L$ & $L$ \\
\hline Autotransplants & & & & & & & & $\begin{array}{l}14 / 19 \mathrm{~L} 5 / \\
19 \mathrm{M}\end{array}$ \\
\hline Kristersson [51] & 1991 & Retrospect & M & L & L & L & $L$ & L \\
\hline Kugelberg [52] & 1994 & ProspObs & M & L & L & $\mathrm{L}$ & $L$ & $L$ \\
\hline Marcusson [53] & 1996 & ProspObs & M & $L$ & L & L & L & L \\
\hline Josefsson [54] & 1999 & Retrospect & M & $\mathrm{L}$ & M & $\mathrm{L}$ & L & $L$ \\
\hline Czochrowska [55] & 2002 & Retrospect & $\mathrm{H}$ & $L$ & M & L & $L$ & M \\
\hline Bauss [23] & 2004 & prospCT & M & $L$ & M & M & M & M \\
\hline Jonsson [56] & 2004 & ProspObs & M & $L$ & L & L & M & $L$ \\
\hline Tanaka [57] & 2007 & Retrospect & M & $L$ & M & L & M & M \\
\hline Mensink [58] & 2010 & Retrospect & M & L & L & L & M & L \\
\hline Kvint [59] & 2010 & Retrospect & M & L & L & $L$ & L & L \\
\hline Bokelund [24] & 2013 & Retrospect & $\mathrm{L}$ & L & $L$ & $L$ & L & $L$ \\
\hline Deciduous teeth & & & & & & & & $\begin{array}{l}8 / 11 \mathrm{~L} 3 / \\
11 \mathrm{M}\end{array}$ \\
\hline Bjerklin [60] & 2000 & ProspObs & M & L & M & L & L & L \\
\hline Ith-Hansen [61] & 2000 & ProspObs & L & L & L & L & L & L \\
\hline Sletten [62] & 2003 & Retrospect & $\mathrm{L}$ & L & M & L & M & L \\
\hline Bjerklin [63] & 2008 & ProspObs & M & L & M & L & L & $\mathrm{L}$ \\
\hline Kjaer [17] & 2008 & Retrospect & L & L & M & $L$ & L & $\mathrm{L}$ \\
\hline Hvaring [16] & 2013 & Cross section & L & L & L & L & L & $L$ \\
\hline
\end{tabular}


Table 2 Summary of the selected studies and quality assessement (Continued)

\begin{tabular}{|c|c|c|c|c|c|c|c|c|}
\hline \multicolumn{4}{|l|}{ Convent. Prosth. } & \multirow[b]{2}{*}{ M } & \multirow[b]{2}{*}{ M } & \multirow[b]{2}{*}{$\mathrm{L}$} & \multirow[b]{2}{*}{ M } & \multirow{2}{*}{$\begin{array}{l}2 / 6 L 4 / 6 M \\
M\end{array}$} \\
\hline Hobkirk [64] & 1989 & Retrospect & $\mathrm{H}$ & & & & & \\
\hline Pröbster [65] & 1997 & Retrospect & M & L & M & L & M & M \\
\hline Garnett [66] & 2005 & Retrospect & M & L & M & L & M & M \\
\hline Dueled [26] & 2008 & Retrospect & M & L & M & L & L & $\mathrm{L}$ \\
\hline Krieger [25] & 2009 & Retrospect & M & L & L & M & M & M \\
\hline Spinas [67] & 2013 & ProspObs & L & L & L & L & M & L \\
\hline & & & & & & & & $2 / 6 \mathrm{~L} 4 / 6 \mathrm{M}$ \\
\hline Total & $n=42$ & $\begin{array}{l}25 \text { retrosp } \\
14 \text { prosp }\end{array}$ & & & & & & $\begin{array}{l}30 / 42 \text { L12/ } \\
42 \mathrm{M}\end{array}$ \\
\hline
\end{tabular}

and confirmed that all implant losses had occurred during healing time before prosthetic restoration. Since healing time was has usually a maximum of 6 months, the follow-up time was set on arbitrary 0.6 year. The study of Durstberger et al. [21] had to be excluded because of vague follow-up data and no information of implant losses during follow-up and most of the mentioned patients were only planned for implant placement but had no implants placed. The studies of Grecchi (a) [22] were pooled with Grecchi (b) [15] for numerical analysis, because of assumed double publication of the same samples. In most of the studies, implants had been placed in conjunction with bone grafting, but only Grecchi (a) [22] had presented outcome data on this aspect. There were no prominent differences for implants placed in grafted bone compared with non-grafted sites. Secondary infraocclusion of approximately $1 \mathrm{~mm}$ was a problem of implant placements in the maxillary incisor region in childhood [3]. Marginal bone resorption at implants was not a prominent finding and ranged from 0.2 to $1.2 \mathrm{~mm}$ in the studies which reported this aspect. The success/survival data and further subgroup analysis for implant survival data is presented below.

\section{Studies on tooth autotransplantation}

A total of 11 studies with a mean follow-up time of 7.6 years (maximum 26.4 years) were included, all of which qualified also for the numerical analysis (Table 4). The study heterogeneity concerning survival and success data was low and acceptable for numerical data analysis. The studies showed some heterogeneity in patient inclusion, because in most studies a tooth agenesis sample was mixed also with missing teeth due to trauma and other reasons, which did not lead to exclusion from the present evaluation. According to the table, three studies found a better survival between 10 and $46 \%$ of immature teeth compared to mature teeth. The Bauss study [23] demonstrated better pulpal and periodontal conditions if a transplantated tooth was not moved orthodontically, in comparison of the different orthodontic treatments derotation was more harmful than extrusion. According to the Bokelund study [24], premolar transplants performed better than molars. A preceding primary tooth at the receptor site of a tooth autotransplant was connected with a lower transplant success than a normal primary tooth. The numerical success/ survival data are presented below.

\section{Studies on preservation of deciduous teeth}

A total of 6 studies with a mean follow-up time of 12.5 years (maximum 16.5 years) was included, of which only 4 qualified for the numerical analysis (Table 5). The studies were homogenous in terms of outcome parameters and inclusion of only tooth agenesis patients. Two studies (Kjaer [17], Hvaring [16] ) contained no data on follow-up times. The Kjaer study made an important statement that in patients in whom the dentitions shows morphological signs of ectodermal dysplasia (screw driver shaper incisors, taurodontism, invaginations of incisors or slim incisors, typical ED sites of aplasia), the risk of root resorption fatal prognosis of a deciduous tooth was elevated by the factor 1.46 compared to patients with normal tooth appearance. In general, root resorption, ankylosis, and consecutive infraocclusion were a problem of preservation of deciduous second molars at the site of a secondary premolar aplasia. The absence of root resorption was interpreted as success criterion in most studies, therefore, in contrast to high survival figures, the success of deciduous teeth was lower. The numerical success/survival data are presented below.

\section{Studies on conventional prosthetics on teeth}

A total of 6 studies with a mean follow-up time of 7.2 years (maximum 15.1 years) was included, all of which qualified for the numerical analysis (Table 6). This relatively small group of studies showed a high level of heterogeneity in patient inclusion, because this group comprised various indications from single missing incisors up to severe hypodontia treated with 
Table 3 Synopsis of included studies on dental implants and prosthetics on dental implants in order of publication year

\begin{tabular}{|c|c|c|c|c|c|c|c|c|c|c|c|c|c|c|}
\hline Author & Year & $\begin{array}{l}\text { Study } \\
\text { type }\end{array}$ & Population & Treatment & Comparison & Patients & Implants & $\begin{array}{l}\text { Implant } \\
\text { survival [\%] }\end{array}$ & $\begin{array}{l}\text { Implant } \\
\text { success [\%] }\end{array}$ & $\begin{array}{l}\text { Prosthes. } \\
\text { survival [\%] }\end{array}$ & $\begin{array}{l}\text { Marginal } \\
\text { resorpt. } \\
{[\mathrm{mm}]}\end{array}$ & $\begin{array}{l}\text { Infra } \\
\text { occlusion } \\
{[\mathrm{mm}]}\end{array}$ & $\begin{array}{l}\text { Follow-up } \\
\text { [y] }\end{array}$ & $\begin{array}{l}\text { Risk of } \\
\text { bias }\end{array}$ \\
\hline Ledermann [40] & 1993 & Retrospect & Agenesis, trauma & FPD & 9-18 years & 34 & 42 & 90 & 90 & 100 & & & 3 & M \\
\hline \multirow[t]{3}{*}{ Kearns [41] } & 1999 & ProspObs & ED 5-17 years & Full denture & All & 6 & 41 & 97.6 & & & & & 7.8 & L \\
\hline & & & & & $<6$ years & 2 & 9 & 100 & 44.4 & 0 & & & 7.8 & \\
\hline & & & & & $>12$ years & 4 & 32 & 96.9 & 96.9 & 100 & & & 7.8 & \\
\hline \multirow[t]{4}{*}{ Thilander [3] } & 2001 & ProspObs & $\begin{array}{l}\text { Agen, }<18 \text { years } \\
\text { trauma }\end{array}$ & SC, FPD & All & 18 & 47 & 100 & 100 & 93.9 & & & 10 & L \\
\hline & & & & & Incisors & 12 & 26 & 100 & 100 & 88.2 & 0.75 & 0.98 & 10 & \\
\hline & & & & & Canines & 4 & 8 & 100 & 100 & 100 & 0.6 & 0 & 10 & \\
\hline & & & & & Premolars & 5 & 13 & 100 & 100 & 100 & 0.5 & $0.1-0.6$ & 10 & \\
\hline \multirow[t]{6}{*}{ Guckes [42] } & 2002 & ProspObs & ED $5-18$ years & Full denture & All & 51 & 264 & 90 & & & & & 2 & L \\
\hline & & & & & Maxilla & & 21 & 71 & & & & & 2 & \\
\hline & & & & & Mandible & & 243 & 91 & & & & & 2 & \\
\hline & & & & & $<11$ years & & 46 & 85 & & & & & 2 & \\
\hline & & & & & $11-18$ years & & 122 & 87 & & & & & 2 & \\
\hline & & & & & $>18$ years & & 96 & 95 & & & & & 2 & \\
\hline \multirow[t]{3}{*}{ Sweeney [43] } & 2005 & Retrospect & ED adolesc. & $\begin{array}{l}\text { FPD, full } \\
\text { denture }\end{array}$ & All & 14 & 61 & 88.5 & & 94 & & & 3.33 & L \\
\hline & & & & & Maxilla & 4 & 15 & 80 & & & & & 3.33 & \\
\hline & & & & & Mandible & 14 & 46 & 91.3 & & & & & 3.33 & \\
\hline \multirow[t]{3}{*}{ Finnema [27] } & 2005 & Retrospect & Oligodont. adults & FPD & All & 13 & 87 & 89.7 & & & & & 3 & $\mathrm{~L}$ \\
\hline & & & & & Maxilla & & & 86 & & & & & 3 & \\
\hline & & & & & Mandible & & & 96 & & & & & 3 & \\
\hline Poggio [44] & 2005 & Retrospect & Oligodont. adults & SC & No & 24 & 46 & 100 & & 82.6 & & & 5.25 & M \\
\hline Zarone [45] & 2006 & ProspObs & Ag incisors adults & SC & No & 30 & 34 & 97.1 & 94.1 & & 1.2 & & 3.75 & L \\
\hline Becelli [46] & 2007 & Retrospect & Oligodont. adults & SC, FPD & No & 8 & 60 & 96.6 & & & & & 8.5 & L \\
\hline \multirow[t]{3}{*}{ Bergendal [20] } & 2008 & Survey & ED, adoles children & n.s. & All & 26 & 47 & 76.6 & & & & & 0.6 & M \\
\hline & & & & & $14-15$ years & 21 & 33 & 93.9 & & & & & 0.6 & \\
\hline & & & & & $<13$ years & 5 & 14 & 35.7 & & & & & 0.6 & \\
\hline Dueled [26] & 2008 & Retrospect & Oligodont & SC, FPD & $\begin{array}{l}\text { Implant } \\
\text { supported }\end{array}$ & 110 & 179 & 100 & 87.3 & 99 & & & 3.8 & L \\
\hline
\end{tabular}


Table 3 Synopsis of included studies on dental implants and prosthetics on dental implants in order of publication year (Continued)

\begin{tabular}{|c|c|c|c|c|c|c|c|c|c|c|c|c|c|}
\hline \multirow[t]{3}{*}{ Krieger [25] } & 2009 & Retrospect & Oligodont. adults & SC, FPD & All impl & 17 & 40 & 92.5 & & 87.9 & & 15.1 & L \\
\hline & & & & & SCImpl & 12 & 24 & 87.5 & 62 & 83.3 & & 15.1 & \\
\hline & & & & & FDP Impl & 6 & 16 & 100 & 100 & 100 & & 15.1 & \\
\hline \multirow[t]{3}{*}{ Degidi [47] } & 2009 & RCT & Ag Incisors adults & SC & All & 60 & 60 & 100 & 98.3 & 100 & & 3 & L \\
\hline & & & & & Imm. load & 30 & 30 & 100 & 100 & 100 & 0.85 & 3 & \\
\hline & & & & & Conv. load & 30 & 30 & 100 & 96.6 & 93.3 & 0.75 & 3 & \\
\hline Creton [1] & 2010 & Retrospect & Oligodont. adults & FPD & No & 44 & 214 & 89.8 & & & & 2.9 & L \\
\hline \multirow[t]{5}{*}{ Grecchi (a) [15] } & 2010 & Retrospect & ED adults & FPD & All & 4 & 44 & 100 & 90.9 & & 0.4 & 1.8 & M \\
\hline & & & & & Grafted & 3 & & 100 & & & 0.3 & & \\
\hline & & & & & Non-graft & 1 & & 100 & & & 1.2 & & \\
\hline & & & & & Maxilla & & 20 & 100 & & & 0.2 & & \\
\hline & & & & & Mandible & & 24 & 100 & & & 0.7 & & \\
\hline $\begin{array}{l}\text { Grecchi (b) } \\
{[22]}\end{array}$ & 2010 & Retrospect & ED adults & FPD & No & 8 & 78 & 98.7 & & & $0.5-0.6$ & 1.8 & Pooled \\
\hline Nissan [48] & 2011 & Unclear & Agenesis & SC & No & 12 & 21 & 95.2 & 95.2 & & & 2.5 & M \\
\hline \multirow[t]{3}{*}{ Heuberer [38] } & 2012 & Retrospect & $\begin{array}{l}\text { Mixed ED }< \\
12 \text { years }\end{array}$ & Full denture & All & 6 & 16 & 93.8 & & 100 & & 4 & L \\
\hline & & & & & $\begin{array}{l}\text { Maxilla, } \\
\text { onplants }\end{array}$ & 4 & 8 & 87.5 & & 100 & & 5 & \\
\hline & & & & & Mandible & 3 & 8 & 100 & & 100 & & 3 & \\
\hline Hosseini [49] & 2013 & ProspObs & Hypodontia & SC & No & 59 & 98 & 100 & 99 & 97.2 & & 3 & L \\
\hline Zou [50] & 2014 & Retrospect & ED Adults & FPD & No & 25 & 169 & 98.3 & 97.2 & 100 & 1.4 & 5 & L \\
\hline
\end{tabular}


Table 4 Synopsis of included studies on tooth autotransplantation in order of publication year

\begin{tabular}{|c|c|c|c|c|c|c|c|c|c|c|c|c|}
\hline Author & Year & Study type & Population & Comparison & Patients & Transplants & $\begin{array}{l}\text { Transplant } \\
\text { survival [\%] }\end{array}$ & $\begin{array}{l}\text { Transplant } \\
\text { success [\%] }\end{array}$ & Pulp vitality [\%] & Intact periodont. [\%] & Follow-up [y] & Risk of bias \\
\hline \multirow[t]{3}{*}{ Kristersson [51] } & 1991 & Retrosp. & Agen, incis, trauma & All & 50 & 50 & 98 & 82 & & & 7.5 & L \\
\hline & & & & Immature & & 41 & & 90 & & & 7.5 & \\
\hline & & & & Mature & & 9 & & 44 & & & 7.5 & \\
\hline \multirow[t]{3}{*}{ Kugelberg [52] } & 1994 & Prosp.Ob & $\mathrm{Ag}, \operatorname{Tr}$ & All & 40 & 45 & 96 & 89 & & & 4 & L \\
\hline & & & & Immature & & 23 & & 96 & 100 & 96 & 4 & \\
\hline & & & & Mature & & 22 & & 82 & 0 & 82 & 4 & \\
\hline Marcusson [53] & 1996 & Prosp.Ob & Ag, missing & No & 29 & 31 & 87 & & & & 8 & L \\
\hline \multirow[t]{3}{*}{ Josefsson [54] } & 1999 & Retrosp. & Oligodontia & All & 80 & 110 & 91 & & & & 4 & L \\
\hline & & & & Immature & & 11 & & 92 & & & 4 & \\
\hline & & & & Mature & & 99 & & 82 & & & 4 & \\
\hline Czochrowska [55] & 2002 & Retrosp. & Agenesis & No & 28 & 33 & 90 & 79 & & & 26.4 & M \\
\hline \multirow[t]{4}{*}{ Bauss [23] } & 2004 & Retrosp. & Ag, missing & All & 88 & 91 & 100 & 84.6 & & & 4 & M \\
\hline & & & & OrthDerot & 27 & 28 & 100 & & 71.4 & 67.8 & 4 & \\
\hline & & & & OrthExtru & 20 & 21 & 100 & & 90.5 & 85.7 & 4 & \\
\hline & & & & NoOrthod & 41 & 42 & 100 & & 97.6 & 95.2 & 4 & \\
\hline Jonsson [56] & 2004 & Prosp.Ob & Ag, missing & No & 32 & 40 & 92.5 & & 76 & 100 & 10 & L \\
\hline Tanaka [57] & 2007 & Retrosp. & Ag, missing & No & 24 & 28 & 100 & & 60.7 & & 4 & M \\
\hline Mensink [58] & 2010 & Retrosp. & Ag, missing & No & 44 & 63 & 100 & & 89 & & 1 & L \\
\hline Kvint [59] & 2010 & Retrosp. & Ag, missing & No & 215 & 215 & 88.4 & 81 & & & 4.8 & L \\
\hline \multirow[t]{5}{*}{ Bokelund [24] } & 2013 & Retrosp. & Agenesis & All & 157 & 211 & 100 & & & & 10 & L \\
\hline & & & & Premolar & & 162 & & 93 & & 93 & 10 & \\
\hline & & & & Molars & & 49 & & 60 & & 60 & 10 & \\
\hline & & & & NormalPrim & & 71 & & 95 & & 95 & 10 & \\
\hline & & & & InfraoccPrim & & 140 & & 87 & & 87 & 10 & \\
\hline
\end{tabular}


Table 5 Synopsis of included studies on preservation of deciduous teeth in order of publication year

\begin{tabular}{|c|c|c|c|c|c|c|c|c|c|c|c|c|}
\hline Author & Year & $\begin{array}{l}\text { Study } \\
\text { type }\end{array}$ & Population & Comparison & Patients & $\begin{array}{l}\text { Deciduous } \\
\text { teeth }\end{array}$ & $\begin{array}{l}\text { Deciduous } \\
\text { tooth } \\
\text { survival } \\
{[\%]}\end{array}$ & $\begin{array}{l}\text { No Infra- } \\
\text { occlusion } \\
\text { (success) } \\
{[\%]}\end{array}$ & $\begin{array}{l}\text { No root } \\
\text { resorption } \\
\text { [\%] }\end{array}$ & $\begin{array}{l}\text { Odds } \\
\text { ratio }\end{array}$ & $\begin{array}{l}\text { Follow-up } \\
\text { [y] }\end{array}$ & $\begin{array}{l}\text { Risk } \\
\text { of } \\
\text { bias }\end{array}$ \\
\hline Bjerklin [60] & 2000 & ProspObs & Agenes & no & 41 & 59 & 88 & 45 & 40 & & 9 & L \\
\hline Ith-Hansen [61] & 2000 & ProspObs & Agenes & no & 18 & 26 & 89.6 & 88.4 & 88.4 & & 16.5 & L \\
\hline Sletten [62] & 2003 & Retrospect & Agenes & No & 20 & 28 & 86 & & & & 12.4 & $\mathrm{~L}$ \\
\hline Bjerklin [63] & 2008 & ProspObs & Agenes & No & 99 & 149 & 91 & 48 & 16.7 & & 12.2 & $\mathrm{~L}$ \\
\hline Kjaer [17] & 2008 & Retrospect & Agenes & $\begin{array}{l}\text { ED shaped } \\
\text { versus normal }\end{array}$ & 105 & n.s. & & & & 1.46 & No & L \\
\hline Hvaring [16] & 2013 & $\begin{array}{l}\text { Cross } \\
\text { section }\end{array}$ & Agenes & No & 111 & 188 & & 57.4 & 66.6 & & No & $L$ \\
\hline
\end{tabular}

overdentures on teeth. Nevertheless, it was decided to keep these results in the numerical analysis, because of relatively consistent success and survival data across the different prosthetic therapies. The numerical success/survival data are presented below.

\section{Data synthesis}

\section{Comparison of the four treatments}

The results of the weighted mean method and annual failure rates are presented in Table 7 . In addition, the results are visualized in Figs. 2, 3, 4, 5, 6, 7, 8, and 9.

There is a marked difference in survival data if implants, autotransplants, and preservation of deciduous teeth are compared with conventional prosthetics on teeth, which has only a prosthesis survival of $60.2 \%$ (CI 9.4) at a mean follow-up of 8.4 years. If not the patient but implants/teeth or prosthetic units are used to weigh the means, the results do not change markedly (Fig. 2).

Looking upon the success data, both deciduous teeth and autotransplants have markedly lower success than survival, which is mainly due to the high rate of ankyloses and root resorption in both treatment options using the natural teeth (Fig. 2).

Implants have higher annual failure rates than the natural tooth alternatives. This is also an effect of the shorter follow-up times in implants (4.6 years) compared to autotransplants (7.6 years) and deciduous teeth (12.5 years) (Fig. 3).

A direct comparison of implant and conventional prosthetics was made in two retrospective studies of Krieger and coworkers [25] and Dueled and coworkers [26]. In both studies, prostheses on implants had better

Table 6 Synopsis of included studies on conventional prosthetics on teeth in order of publication year

\begin{tabular}{|c|c|c|c|c|c|c|c|c|c|c|c|c|}
\hline Author & Year & $\begin{array}{l}\text { Study } \\
\text { type }\end{array}$ & Population & Treatment & Comparison & Patients & Teeth & Prostheses & $\begin{array}{l}\text { Prosthesis } \\
\text { survival } \\
\text { [\%] }\end{array}$ & $\begin{array}{l}\text { Prosthesis } \\
\text { success } \\
\text { [\%] }\end{array}$ & $\begin{array}{l}\text { Follow-up } \\
\text { [y] }\end{array}$ & $\begin{array}{l}\text { Risk } \\
\text { of } \\
\text { bias }\end{array}$ \\
\hline \multirow[t]{3}{*}{ Hobkirk [64] } & 1989 & Retrospect & Hypodontia & Overdenture & All & 138 & & 138 & 51.5 & & 6 & $M$ \\
\hline & & & & & Mandible & 48 & & 48 & 33 & & 6 & \\
\hline & & & & & Maxilla & 90 & & 90 & 61 & & 6 & \\
\hline Pröbster [65] & 1997 & Retrospect & HypodTrauma & Single crown & No & 264 & 392 & 325 & 60 & & 10 & M \\
\hline Garnett [66] & 2005 & Retrospect & Hypodontia & $\begin{array}{l}\text { Single incis } \\
\text { crown }\end{array}$ & & 45 & 73 & 73 & 59 & & 6 & M \\
\hline \multirow[t]{2}{*}{ Dueled [26] } & 2008 & Retrospect & $\begin{array}{l}\text { Oligodont } \\
\text { adults }\end{array}$ & SC, FPD & On teeth & 19 & & 30 & 89.5 & 80 & 3.8 & L \\
\hline & & & & SC, FPD & On implants & 110 & & 179 & 99 & 91 & 3.8 & \\
\hline \multirow[t]{6}{*}{ Krieger [25] } & 2009 & Retrospect & Hypodontia & All & On teeth & 5 & & 25 & 85.7 & 42 & 15.1 & M \\
\hline & & & & Single $\mathrm{Cr}$. & On teeth & 5 & & 5 & 80 & 80 & 15.1 & \\
\hline & & & & FDP & On teeth & 10 & & 20 & 84.1 & 72.8 & 15.1 & \\
\hline & & & & All & On implants & 17 & & 33 & 87.9 & 53 & 15.1 & \\
\hline & & & & SC & On implants & 12 & & 24 & 83.3 & 62.5 & 15.1 & \\
\hline & & & & FDP & On implants & 6 & & 9 & 100 & 66.7 & 15.1 & \\
\hline Spinas [67] & 2013 & Prosp.Obs. & Hypodontia & $\begin{array}{l}\text { Resin bridge } \\
\text { single Incis }\end{array}$ & & 30 & 32 & 32 & 93.8 & & 5 & L \\
\hline
\end{tabular}


Table 7 Numerical results of general outcome parameters (weighted mean values)

\begin{tabular}{|c|c|c|c|c|c|c|c|c|c|c|c|c|c|c|c|c|}
\hline [\%] & $\begin{array}{l}\text { Survival } \\
\text { patient } \\
\text { weighted }\end{array}$ & $\begin{array}{l}95 \% \\
\text { confid. } \\
\text { interval }\end{array}$ & $\begin{array}{l}\text { Success } \\
\text { patient } \\
\text { weighted }\end{array}$ & $\begin{array}{l}95 \% \\
\text { confid. } \\
\text { interval }\end{array}$ & $\begin{array}{l}\text { Survival } \\
\text { impl/tooth/ } \\
\text { unit weighted }\end{array}$ & $\begin{array}{l}95 \% \\
\text { confid. } \\
\text { interval }\end{array}$ & $\begin{array}{l}\text { Success } \\
\text { impl/tooth/ } \\
\text { unit weighted }\end{array}$ & $\begin{array}{l}95 \% \\
\text { confid. } \\
\text { interval }\end{array}$ & $\begin{array}{l}\text { Annual } \\
\text { failure rate } \\
\text { survival } \\
\text { patient } \\
\text { weighted }\end{array}$ & $\begin{array}{l}95 \% \\
\text { confid. } \\
\text { interval }\end{array}$ & $\begin{array}{l}\text { Annual } \\
\text { failure rate } \\
\text { success } \\
\text { patient } \\
\text { weighted }\end{array}$ & $\begin{array}{l}95 \% \\
\text { confid. } \\
\text { interval }\end{array}$ & $\begin{array}{l}\text { annual failure } \\
\text { rate survival } \\
\text { impl/tooth/ } \\
\text { unit weighted }\end{array}$ & $\begin{array}{l}95 \% \\
\text { confid. } \\
\text { interval }\end{array}$ & $\begin{array}{l}\text { Annual failure } \\
\text { rate success } \\
\text { impl/tooth/ } \\
\text { unit weighted }\end{array}$ & $\begin{array}{l}95 \% \\
\text { confid. } \\
\text { interval }\end{array}$ \\
\hline \multicolumn{17}{|c|}{ Treatment comparisons } \\
\hline Implants & 95.3 & 1.9 & 92.7 & 4.6 & 94.1 & 1.8 & 95.6 & 4.1 & 3.317 & 0.196 & 0.815 & 0.055 & 3.280 & 0.150 & 1.987 & 0.159 \\
\hline AutoTX & 94.4 & 4.1 & 82.5 & 11.7 & 94.8 & 4.0 & 85.0 & 4.0 & 1.061 & 0.112 & 1.832 & 0.638 & 1.167 & 0.100 & 2.334 & 0.184 \\
\hline Deciduous & 89.6 & 19.1 & 51.8 & 12.6 & 89.7 & 19.8 & 51.7 & 13.1 & 0.908 & 0.157 & 2.928 & 1.472 & 0.903 & 0.161 & 4.333 & 1.494 \\
\hline ConvProsth & 60.2 & 9.4 & 59.4 & 44.3 & 62.6 & 7.6 & 59.4 & 44.3 & 5.144 & 0.851 & 5.368 & 4.947 & 11.098 & 1.477 & 9.863 & 6.895 \\
\hline \multicolumn{17}{|c|}{ Implant subgroup analysis } \\
\hline Children & 72.4 & 18.8 & 44.4 & n.a. & 80.1 & 17.6 & 44.4 & n.a. & 50.177 & 32.083 & 7.128 & n.a. & 25.532 & 9.836 & 7.128 & n.a. \\
\hline Adolescents & 93.0 & 9.5 & 93.7 & 27.0 & 82.0 & 8.3 & 95.7 & 7.5 & 4.610 & 1.029 & 2.052 & 1.313 & 4.626 & 0.712 & 1.262 & 0.724 \\
\hline Adults & 97.4 & 4.0 & 92.2 & 7.5 & 95.6 & 3.3 & 91.4 & 6.8 & 0.670 & 0.001 & 1.850 & 0.272 & 1.280 & 0.122 & 2.188 & 0.276 \\
\hline Ectod.Dyspl. & 89.6 & 8.4 & 93.4 & 30.3 & & & & & 11.665 & 2.520 & 1.431 & 0.219 & & & & \\
\hline Hypo/oligodo & 97.2 & 3.9 & 92.4 & 5.4 & & & & & 0.864 & 0.067 & 1.899 & 0.199 & & & & \\
\hline $\begin{array}{l}\text { Prosthesis on } \\
\text { implants }\end{array}$ & 97.8 & 2.3 & 94.5 & 2.4 & & & & & 0.864 & 0.032 & 0.884 & 0.029 & & & & \\
\hline $\begin{array}{l}\text { Prosthesis on } \\
\text { teeth }\end{array}$ & 61.4 & 7.9 & 77.9 & 19.2 & 62.1 & 7.7 & 77.4 & 20.6 & 5.060 & 0.726 & 3.666 & 1.695 & 12.111 & 1.496 & 3.046 & 1.650 \\
\hline Sing. crowns & 98.5 & 24.5 & & & & & & & & & & & & & & \\
\hline FPD & 96.3 & 7.7 & & & & & & & & & & & & & & \\
\hline Full dentures & 90.6 & 9.0 & & & & & & & & & & & & & & \\
\hline Maxilla & & & & & 84.2 & 8.3 & & & & & & & & & & \\
\hline Mandible & & & & & 91.9 & 30.3 & & & & & & & & & & \\
\hline Single aplas & 99.1 & 14.5 & & & & & & & & & & & & & & \\
\hline Mild hypodo & 94.6 & 5.3 & & & & & & & & & & & & & & \\
\hline Severe Oligo & 93.1 & 11.0 & & & & & & & & & & & & & & \\
\hline
\end{tabular}



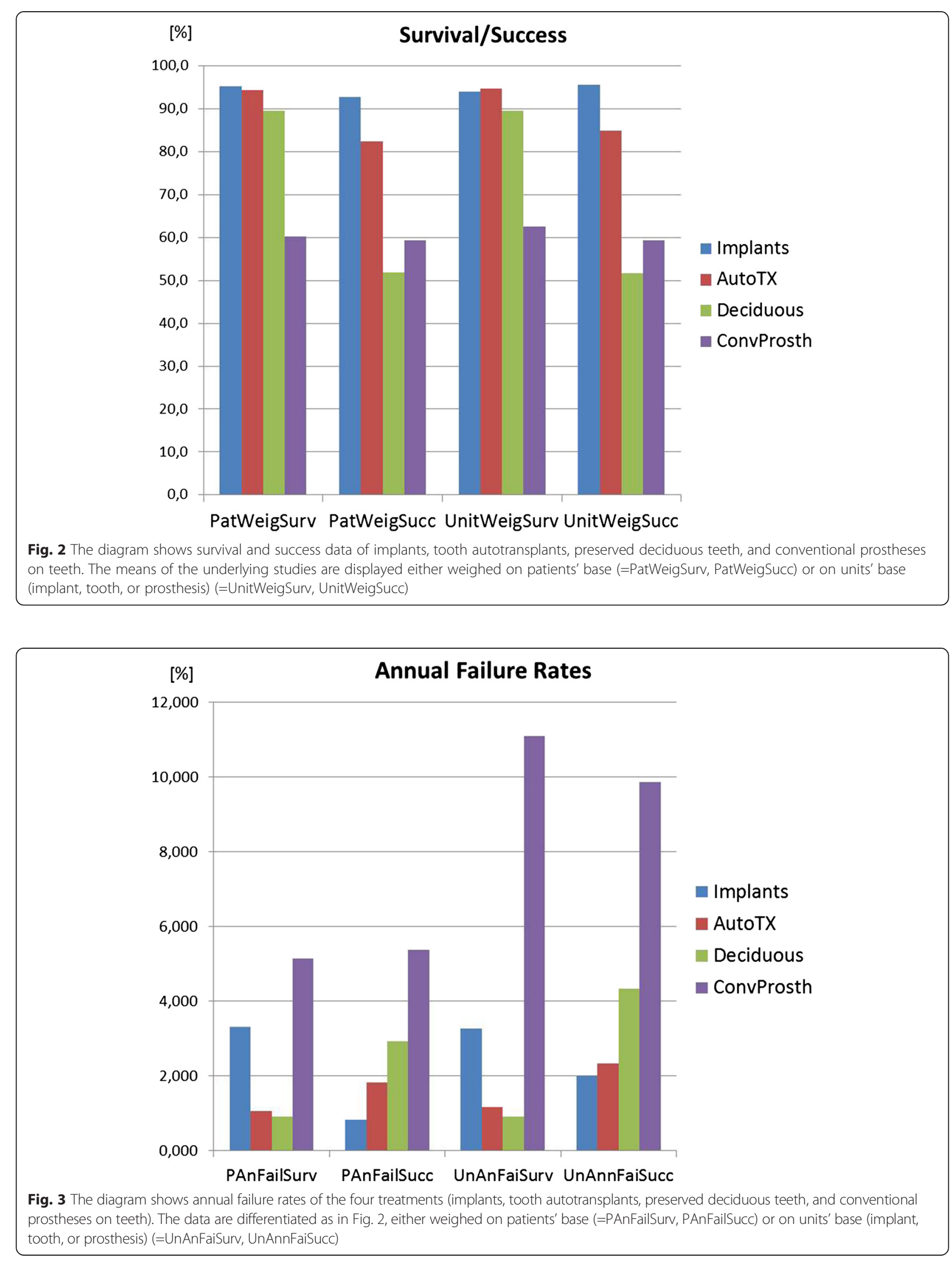

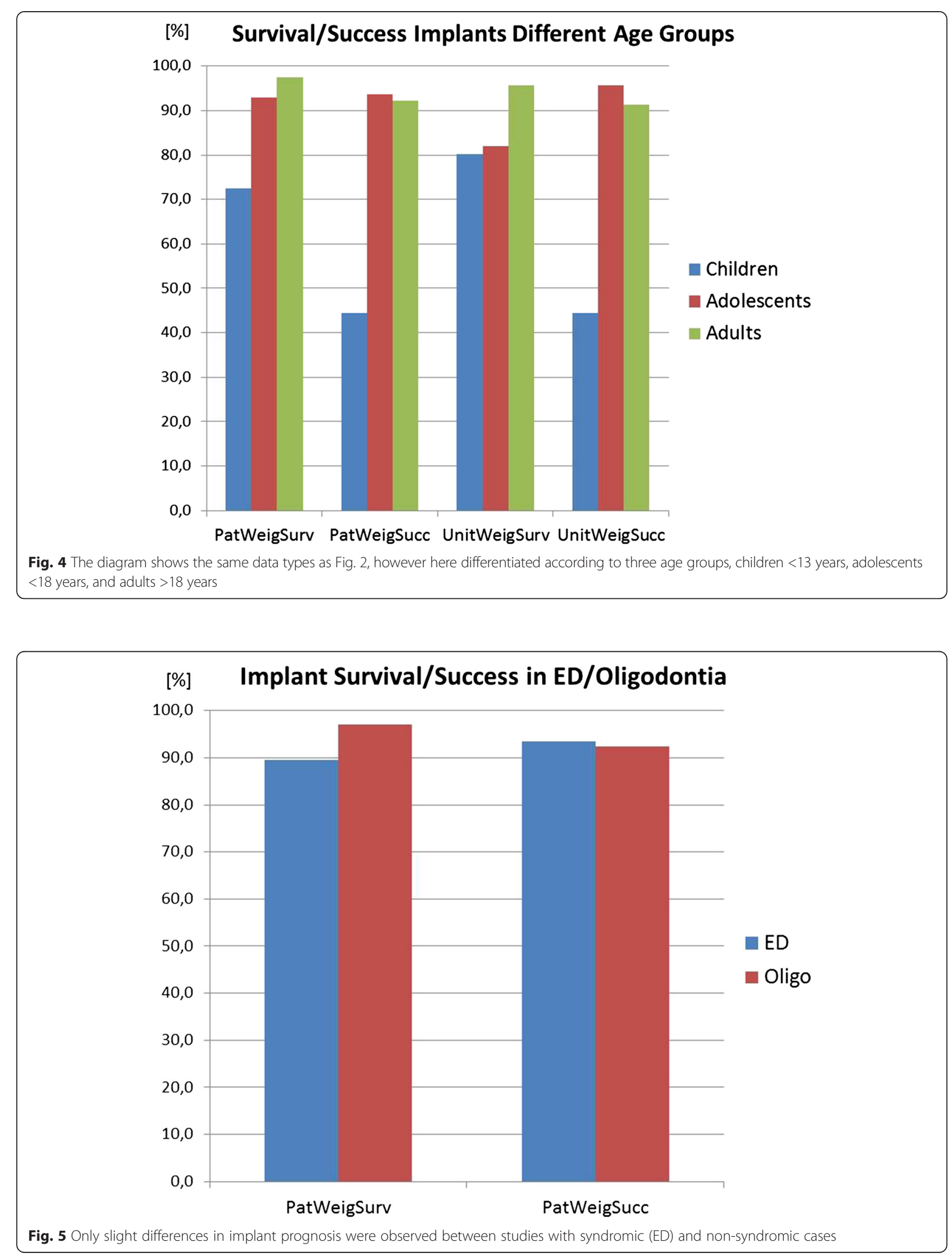

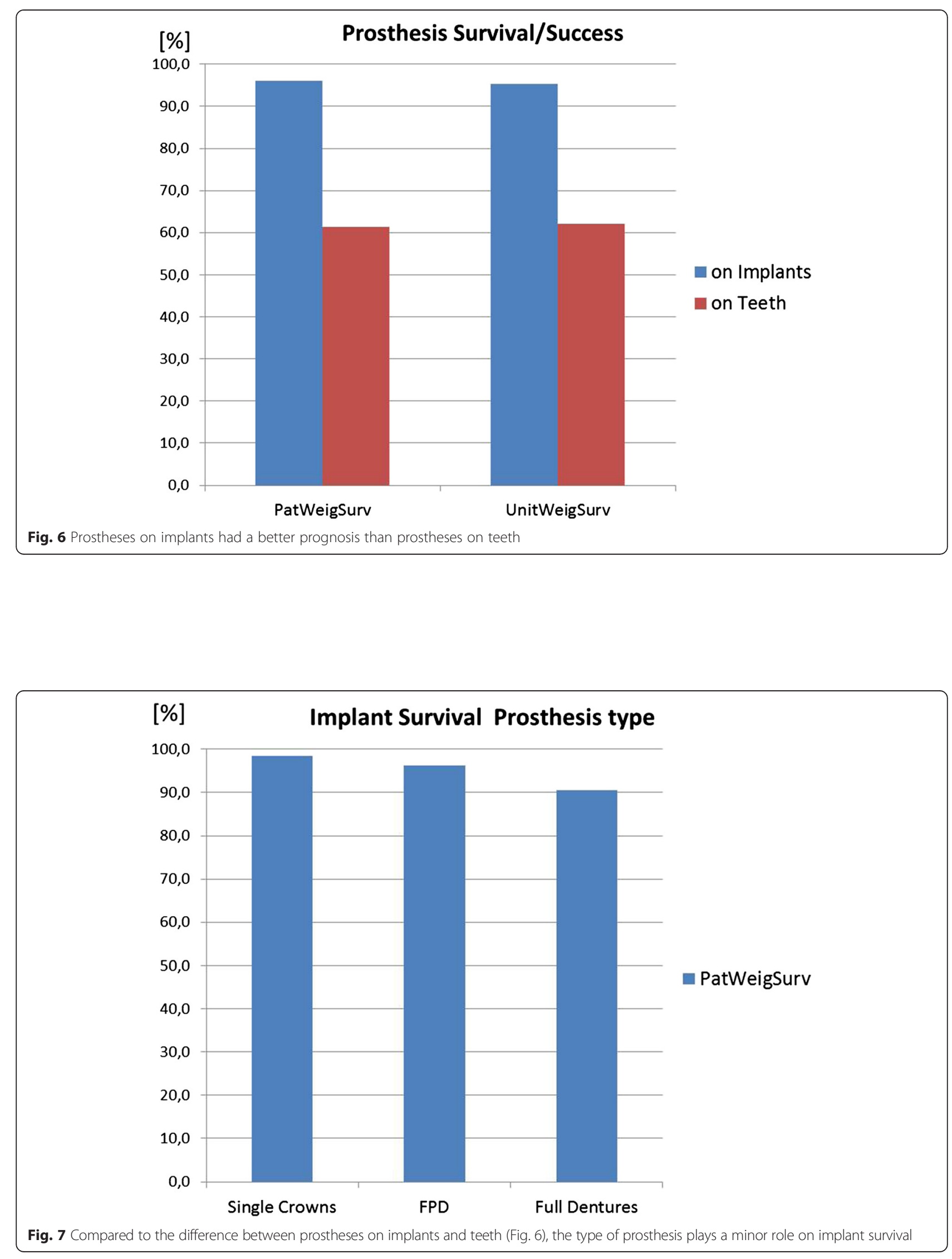


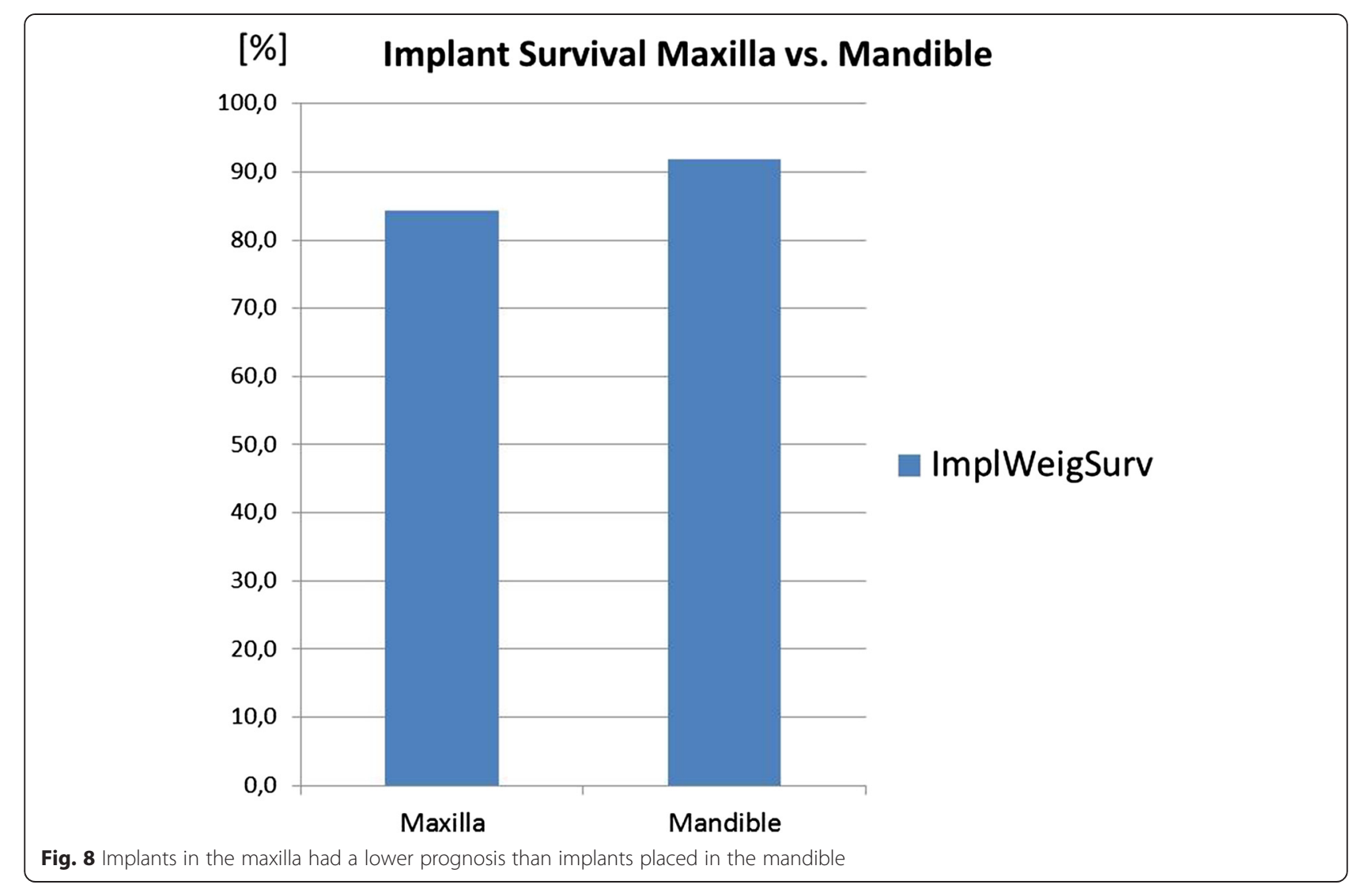

survival than on teeth. In the Dueled study, the difference of the prosthesis survival rates between implants and teeth was $9.5 \%$ and the success rates differed by $11 \%$ in favor of implants. The respective differences in the Krieger study were a survival difference of $2.2 \%$ and a prosthesis success difference of $11 \%$. Krieger and coworkers observed also marked differences of $>40 \%$ between prosthesis success and survival, as an indicator for prosthetic maintenance and repair needs over a very long follow-up time of 15.1 years.

\section{Subgroup analysis of dental implant treatment Children versus adolescents versus adults}

There is a clearly lower implant survival with $72.4 \%$ (CI 18.8) when dental implants are used in childhood below the age of 13 (Fig. 4). In most included studies, implant losses in children occurred early during the healing phase. Also in adolescents below the age of 18 years with a success of $93.0 \%$ (CI 9.5) dental implants performed slightly lower than in adults with a success of $97.4 \%$ (CI 4.0) (Fig. 3). The annual implant failure rates in children were $50.177 \%$ (CI 32.083), in adolescents $4.610 \%$ (CI 1.029 ) and in adults $0.670 \%$ (CI 0.001) (Table 7). The mean observation time of the here included studies in children was 4.1 year, in adolescents 4.9 years, and in adults with congenitally missing teeth 6.4 years.

\section{ED versus non-syndromic congenitally missing teeth (oligodontia)}

The implant success/survival data of ED patients are slightly lower than of non-syndromic patients (Fig. 5). The difference is more marked looking upon annual failure rates (Table 7), indicating that these losses occurred after short observation times. These losses were observed mainly during healing time in children suffering from ED.

\section{Prosthesis survival on teeth versus implants}

The prosthesis survival on teeth is $61.4 \%$ (CI 7.9) after a mean observation time of 7.2 years. On implants, this figure is markedly higher with $97.8 \%$ (CI 2.3) prosthesis survival after a mean observation of 4.6 years (Fig. 6).

\section{Prosthesis type}

The survival data of implants restored with single crowns are slightly higher than restored with fixed partial dentures than restore with full dentures (Fig. 7).

\section{Maxilla versus mandible}

The mandible shows with $91.9 \%$ (CI 30.3) a better implant prognosis in patients with congenitally missing teeth than the maxilla with $84.2 \%$ (CI 8.3) (Fig. 8). 


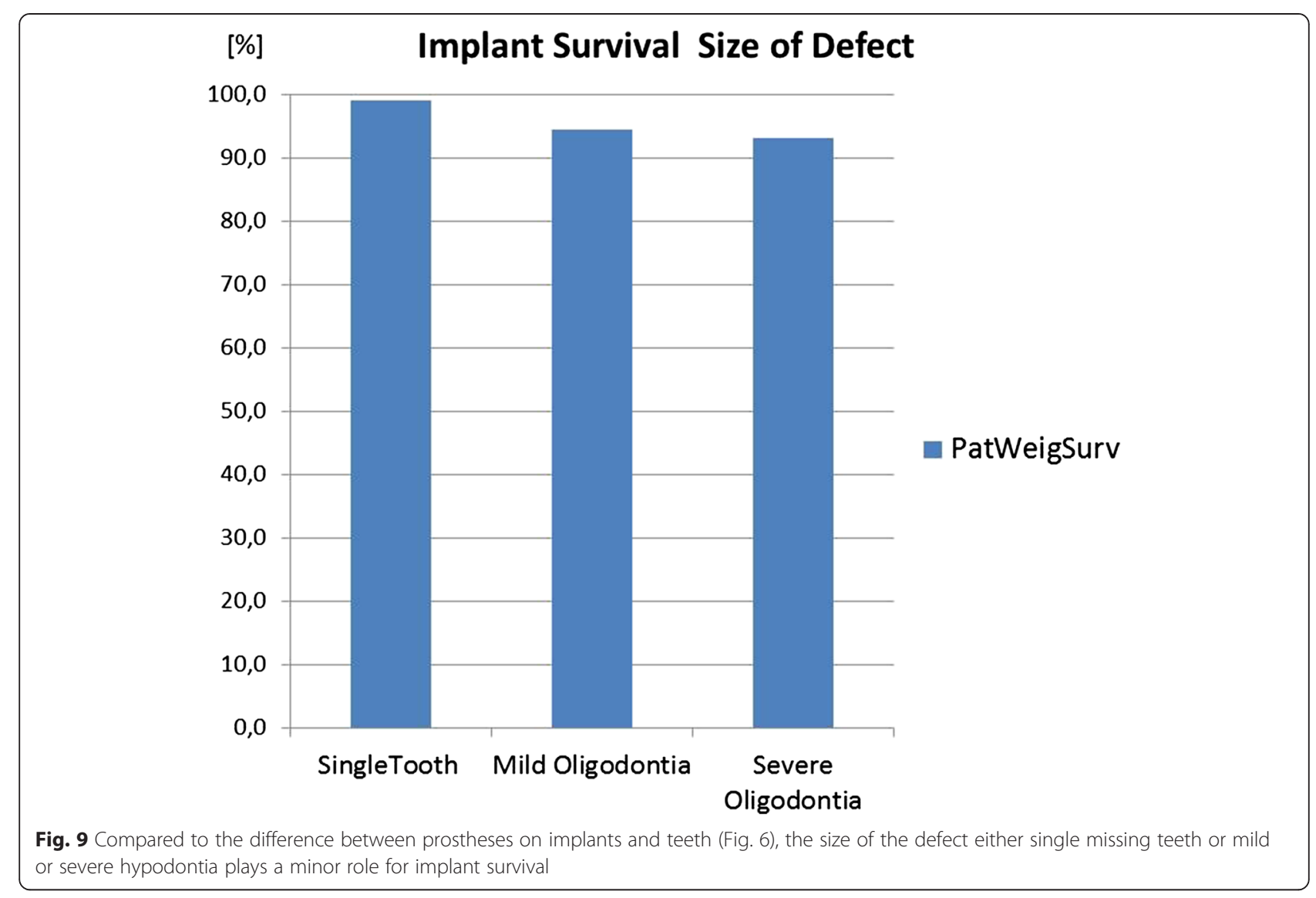

\section{Size of defect}

Patients with single tooth aplasias (99.1\% (CI 14.5)) had better implant survivals than patients with mild hypodontias (94.6 (CI 5.3) and patients with severe hypodontias (93.1 (CI 11.0)) (Fig. 9).

\section{Studies on patient-centered outcome parameters Studies on quality of life, self-esteem, and patient satisfaction}

A total of 16 retrieved studies included numerical assessment data of patient-centered parameters (Table 8). Many studies were cross-sectional studies with description of a baseline situation in hypodontia patiendraw 1 ts. The patient inclusion was relatively homogenous between the studies. However, the studies were relatively heterogenous in the outcome parameters and study treatments because implant, conventional prosthetism, autotransplants, and orthodontic gap closure were included in this table. The number of studies in each treatment was in some cases $n=1$ making numerical comparisons difficult.

Nevertheless, a numerical evaluation using the weighted mean method of the parameters OHIP49 (Oral Health Impact Profile), CPQ11-14 (Child Perceptions
Questionnaire), and patient satisfaction was performed. The results are displayed in Table 9. The mean baseline score of the Oral Health Impact Profile 49 in prosthetically not treated adults was 27.8 (CI 0.9 ) points of a possible maximum of 196 points (14.1\% of maximum) indicating that the patients were not strongly limited by the disease. The baseline scores of the Children Perceptions Questionnaire 11-14 in untreated children with oligodontia was 26.2 (CI 2.2) of 148 maximum score points (17.7 \% of maximum). A mean improvement of 14.9 OHIP points after prosthetic treatment and occlusal rehabilitation was calculated from three reporting studies. The reported improvement with conventional prosthetics was 19.5 points (only one study), with implant prosthetics 12.5 score points (only two studies). Patient satisfaction rates after treatment was $66.5 \%$ (one study) with orthodontic space closure $75 \%$ in autotransplants (one study), $76.6 \%$ with conventional prosthetics (two studies), and $93.4 \%$ with implant prosthetics (3 studies) in hypodontia patients (Table 9).

Two assessments of self-esteem were available. Hashem found self-esteem not significantly affected in hypodontia patients compared to normal control patients. Finnema observed an improvement of patients' 
Table 8 Synopsis of included studies on patient centered outcome parameters in order of publication year Autor Year Study type Population Treatment Comparison Patients Implants/ Baseline OHIP $\begin{array}{llllll}\text { OHIP } & \text { Rosenberg } & \text { Self-esteem } & \text { CPQ } & \text { Satisfact } & \text { Risk } \\ \text { esthetic } & \text { self-esteem } & \text { improvement } & {[\text { scores }]} & \text { [\% sample } & \text { of } \\ \text { [scores] } & {[\text { scores] }} & {[\%]} & & \text { or vas [\%] } & \text { bias }\end{array}$

\begin{tabular}{lll}
\hline Marcusson 1996 Retrospective Agenesis missing \\
[53]
\end{tabular}
missing [scores] [scores] 
Table 8 Synopsis of included studies on patient centered outcome parameters in order of publication year (Continued)

\begin{tabular}{|c|c|c|c|c|c|c|c|c|c|c|c|}
\hline \multirow[t]{2}{*}{$\begin{array}{l}\text { Anweigi (a) } \\
\text { [74] }\end{array}$} & 2013 & $\begin{array}{l}\text { Long Prosp } \\
\text { Obs }\end{array}$ & Hypodontia & $\begin{array}{l}\text { Bridges } \\
\text { orthod. }\end{array}$ & $\begin{array}{l}\text { FPD } \\
\text { completed }\end{array}$ & 40 & n.g. & 35 & -19.5 & & L \\
\hline & & & & & $\begin{array}{l}\text { Orthodontic } \\
\text { phase }\end{array}$ & 37 & n.g. & 32 & 22 & & \\
\hline \multirow{2}{*}{$\begin{array}{l}\text { Anweigi (b) } \\
\text { [75] }\end{array}$} & 2013 & Cross section & NonsyndrOligodonti & n.g. & $16-18$ years & 40 & n.g. & 28 & & & L \\
\hline & & & & & 19-34 years & 42 & n.g. & 33.5 & & & \\
\hline Zou [50] & 2014 & Retrospective & ED & Implants & No & 25 & 169 & & & 91 & M \\
\hline
\end{tabular}


Table 9 Numerical results of patient-centered outcome parameters (weighted mean values)

\begin{tabular}{|c|c|c|c|c|c|c|c|c|}
\hline OHIP & Baseline OHIP & $\begin{array}{l}95 \% \text { confid. } \\
\text { interval }\end{array}$ & $\begin{array}{l}\text { OHIP } \\
\text { improvement } \\
\text { general }\end{array}$ & $\begin{array}{l}95 \% \text { confid. } \\
\text { interval }\end{array}$ & $\begin{array}{l}\text { OHIP } \\
\text { improvement } \\
\text { implants }\end{array}$ & $\begin{array}{l}95 \% \text { confid. } \\
\text { interval }\end{array}$ & $\begin{array}{l}\text { OHIP improvement } \\
\text { conventional }\end{array}$ & $\begin{array}{l}95 \% \text { confid } \\
\text { interval }\end{array}$ \\
\hline [Score units] & 27.8 & 0.9 & 14.9 & 1.7 & 12.5 & 0.2 & 19.5 & n.a. \\
\hline CPQ & Baseline CPQ & & & & & & & \\
\hline [Score units] & 26.6 & 2.2 & & & & & & \\
\hline $\begin{array}{l}\text { Patient } \\
\text { satisfaction }\end{array}$ & $\begin{array}{l}\text { Satisfaction } \\
\text { orthod. clos }\end{array}$ & & $\begin{array}{l}\text { Satisfaction } \\
\text { AutoTX }\end{array}$ & & $\begin{array}{l}\text { Satisfaction } \\
\text { conventional }\end{array}$ & & $\begin{array}{l}\text { Satisfaction } \\
\text { implants }\end{array}$ & \\
\hline [\%] & 66.5 & n.a. & 75.0 & n.a. & 76.6 & 5.2 & 93.4 & 19.3 \\
\hline
\end{tabular}

self-esteem in $61 \%$ of cases after treatment with dental implants (Table 9).

\section{Studies on chewing efficacy}

Two studies were retrieved on chewing efficacy data after prosthetic treatment of hypodontia with implants. Both studies used different methods of assessment. In the study of Finnema and coworkers [27], the mandibular function impairment questionnaire (MFIQ) dropped from 2.23 (44.6\% of maximum scoring range of 5) at baseline to 0.31 after occlusal rehabilitation. The study of Goshima and coworkers [28] reported a marked improvement of bite force, masticatory index and functional impairment index, reduced chewing time, and increased occlusal contact area after prosthetic treatment with dental implants (Table 10).

\section{Further studies}

\section{Studies on orthodontic treatments in patients with} congenitally missing teeth

The literature search according to the applied criteria revealed four relevant studies on orthodontic therapy. Since orthodontic therapy is in most cases a supportive therapy and no competing therapy orthodontics were not included into the numerical evaluation. Robertsson and Mohlin [29] compared directly in a cross-sectional study of 50 patients with missing lateral incisors, orthodontic space closure with orthodontic gap opening, and a bridge. The patients were slightly more satisfied with the esthetics of the space closure. There were slight differences in general patient satisfaction (see there) in favor of the bridge and no differences in any functional parameters. A remarkable finding in two studies of Uribe and coworkers [30] was that after orthodontic opening of a gap of an agenesis of a lateral incisor, the alveolar ridge lost $1.6 \mathrm{~mm}$ ridge width and $0.6 \mathrm{~mm}$ ridge height, measured retrospectively in stone models of 31 patients. In their second study [31], the same problem was measured with cone beam CT and a shrinkage of the width of the alveolar of $0.9 \mathrm{~mm}$ and no height reduction was measured in 11 patients. Dueled [26] observed in severe hypodontia the patients' root resorption in $36 \%$ of oligodontia cases, who received adjunctive orthodontic therapy, which was not found in patients without orthodontic therapy. According to the authors, possible explanations for this finding may have been narrower and more cortical alveolar ridges in edentulous sites of tooth agenesis patients and different tooth root morphology (taurodontism).

\section{Studies on craniofacial growth}

In this group, 3 studies were retrieved according to the search criteria. The two studies of Dellavia and coworkers $[18,19]$ contained drawings (polar diagrams) but not the underlying numerical data. Their evaluation of facial photographies demonstrated that patients with ectodermal dysplasia had a slightly reduced global facial growth in comparison with normal reference peers, with a delay of about 2 years in mandibular and maxillary peak developments. The cross-sectional study of Johnson and coworkers [32] analyzed lateral cephalograms of 50 ED patients without treatment with dental implants and compared them with 45 ED patients, who had received dental implants. Craniofacial morphology did not differ significantly between implant-treated and non-treated ED patients.

\section{Discussion}

This systematic review of the literature made a few noticeable findings. Dental implants and implant-borne prostheses demonstrated high survival and success data with approximately $30 \%$ higher survival/success figures than conventional prostheses on teeth. However, in children implant and prosthesis, success was $20 \%$ lower and success $40 \%$ lower than in adults and adolescents. The factor severity of hypodontia, syndromal versus non-syndromal tooth defects, size and type of the prosthesis, maxilla versus mandible, were in comparison to the age group of minor significance for the implant prognosis. The two other non-prosthetic treatment options using natural teeth, tooth autotransplants, and preservation of the 
Table 10 Synopsis of studies of chewing performance before and after treatment with dental implants

\begin{tabular}{|c|c|c|c|c|c|c|c|c|c|c|c|c|c|c|}
\hline Autor & Year & Study type & Population & Treatment & Comparison & Patients & $\begin{array}{l}\text { Replaced } \\
\text { teeth }\end{array}$ & Bite force $[\mathrm{N}]$ & $\begin{array}{l}\text { Color change } \\
\text { chew.gum [a*] }\end{array}$ & $\begin{array}{l}\text { Chewing } \\
\text { time }[s]\end{array}$ & $\begin{array}{l}\text { Occlusal contact } \\
\text { area }\left[\mathrm{mm}^{2}\right]\end{array}$ & $\begin{array}{l}\text { Masticatory i } \\
\text { ndex 0-3 }\end{array}$ & MFIQ & Study quality \\
\hline \multirow[t]{2}{*}{ Finnema [27] } & 2005 & Retrospect. & Oligodontia & Implant FPD & $\begin{array}{l}\text { Before } \\
\text { treatment }\end{array}$ & 13 & 156 & & & & & & 2.23 & $M$ \\
\hline & & & & & $\begin{array}{l}\text { After } \\
\text { treatment }\end{array}$ & & & & & & & & 0.31 & \\
\hline \multirow[t]{2}{*}{ Goshima [28] } & 2009 & ProspObs & Oligodontia & $\begin{array}{l}\text { Implant sing. } \\
\text { crown }\end{array}$ & $\begin{array}{l}\text { Before } \\
\text { treatment }\end{array}$ & 18 & 37 & 1087.3 & 18.8 & 21.1 & 34.3 & 0.3 & & L \\
\hline & & & & & $\begin{array}{l}\text { After } \\
\text { treatment }\end{array}$ & & & 1383 & 23.2 & 17.9 & 44.9 & 0 & & \\
\hline
\end{tabular}


deciduous teeth had survival rates in the range of dental implants but lower success rates due to a considerable incidence of ankylosis, root resorption, and infraocclusion. Also, patient satisfaction rates were higher for dental implants compared to the other treatment options.

According to the present data, dental implants in patients with congenitally missing teeth have an excellent documented prognosis with survival rates of $95.3 \%$ after a mean follow-up of 4.6 years. The prognosis even rises to $97.4 \%$, if only adults are considered taking into account the higher failure rates in children as discussed below. These figures are well lined with currently published data on implant prognosis in conventional dental implant patients. For example, a 95.5-96.3 \% 5-year survival rate has been published in a recent meta-analysis [33].

The pictures changes slightly in favor of autotransplants and preservation of deciduous teeth when annual failure rates are calculated by dividing the survival/success data through the years of observation. Still, conventional prosthetics have the highest annual failure rates with approximately $5 \%, 5$ times higher than the annual failure rates after tooth autotransplantation and preservation of natural teeth with approximately $1 \%$. The annual failure rate is first of all the mathematical result of the 2-3 times longer observation time in the included studies on the treatment options using natural teeth. On the other hand, this finding confirms the clinical experience that a once healed autotransplant or a once preserved deciduous tooth has in contrast to prosthetic components causes hardly any maintenance and repair expenditures. The latter problem is even more evident, if in the present data, the annual survival-based failure rate of $5 \%$ for conventional prosthetics is compared with the annual success-based failure rate of conventional prosthetics of $11 \%$. Survival means that the prosthetic component is still in the oral cavity whereas success can be much lower due to prosthetic complications and treatment needs. Here, the presented $3 \%$ annual failure rate for dental implants ranges in the middle between conventional prosthetics and treatments with natural teeth. Again, this figure is pretty much in line with recent findings on annual failure rates in implants in conventional dental implant patients. A recently published metaanalysis on implants in conjunction with sinus floor augmentations reported an annual failure rate of $3.5 \%$ [34].

The low prognosis of dental implants in children (72.4 \%) compared to adolescents $(93.0 \%)$ and adults (97.4\%) was not surprising. Also, the systematic review of Yap and Klineberg [11] came to this conclusion. But the large difference of $25 \%$ was remarkable and is clinically relevant. The high annual failure rates of implants in children according to the included studies of $50.2 \%$ compared to $4.6 \%$ in adolescents and $0.7 \%$ in adults with congenitally missing teeth can be alarming. This is in part again an effect of the lower observation times in the included studies (factor 1.5). At this point, also the inclusion of the observation of Bergendal and coworkers [20] has to be discussed, who observed in a survey in Swedish specialist dental clinics an implant loss rate of 6.1 in adolescents and $64.3 \%$ in children under the age of 13 . She reported only healing failures of the implants and no long-term problems in children. The data have been included in this systematic review of the literature here, although it is not a true clinical study and the observation time of 6 months is arbitrary and short. The relevance of the observation of Bergendal has already been discussed elsewhere in the literature [35]. The study was also included because the finding fits to other studies in this review. A biological explanation of healing problems of dental implants in young children may be the brittle cortical bone structure and a more active immune system in children compared to adults and adolescents. An international Delphi consensus group, too, did not reach consensus on the use of dental implants in growing children affected by hypodontia [9, 10]. Decision making for dental implants in children and adolescents cannot only be based on survival data. It includes also secondary infraocclusion of restorations on implants which can account for the upper incisor region up to $2.2 \mathrm{~mm}$ [3]. Less infraocclusion had been observed for teeth in the lower jaw and upper canines [3].

According to the setting of the second consensus conference of the German Implant Association, in this systematic review, special emphasis had to be laid on patient-reported and patient-centered outcomes. In summary, the retrieved studies showed that patients with hypodontia are less disabled than expected, as demonstrated by the moderate OHIP and CPQ scores. For example, according to a study in edentulous wearers of full dentures before implant stabilization, the baseline OHIP score was much higher (54.2) [36], compared to a mean baseline score in the studies included here of 27.8. This observation may be explained by the adaptation of the juvenile hypodontia patients to the situation from early childhood. Patients do not know it differently. This has also an impact on measurements of self-esteem, which according to Hashem and coworkers was not significantly different between hypodontia patients and control patients [37]. Nevertheless, an effect of occlusal rehabilitation was measurable with quality of life data in three available studies on that topic in the present data. Obviously, there is a lack of clinical studies using quality of life data in the field of congenitally missing teeth. Due to heterogeneity and low number of studies, data with patient-reported outcomes in this review have to be interpreted with caution. The same restrictions apply to interpretations of the presented data on the effect of occlusal rehabilitation on masticatory performance in hypodontia patients. 
The PICO question asked whether an early occlusal rehabilitation with dental implants in comparison to tooth autotransplants, conventional prosthetics on teeth, or preservation of deciduous teeth has a better outcome. Based on the presented data here, the question can be answered with yes.

However, each treatment has its time. Preservation of deciduous teeth and autotransplantation is an ideal option in children and adolescents, when dental implants have reduced success rates. The latter option can also be used as a temporary solution until completion of growth. As shown here by the OHIP and self-esteem data and facial growth data, patients with severe oligodontia benefit from early occlusal rehabilitation. A practicable way to safe application of implants in children affected by severe oligodontia may be the proposal by Heuberer and coworkers [38], who used with good success onplants in the maxilla placed in the palate behind the teeth to fix an overdenture prosthesis. In this region, less infraocclusion and less interference with transversal palatal growth are expected. Accordingly for the same reasons, implants can be placed in the mandibular canine region. Also, costs play a role in clinical decision-making with tooth autotransplantation being the most cost-effective option [39] along with preservation of primary teeth, which virtually causes no costs. If the costs are manageable, in clinical decision-making, conventional tooth-borne prosthetic solutions should be thoroughly weighted against implants.

\section{Conclusion}

In synopsis of general and patient-centered outcomes, implants yielded the best results, however, not in children younger than 13 years. Autotransplants and deciduous teeth had low annual failure rates and are appropriate treatments in children and adolescents at low costs. Conventional prosthetics had lower survival/ success rates than the other options. Due to heterogeneity and low number of studies, patientreported outcomes in this review have to be interpreted with caution.

\section{Competing interests}

HT received lecture fees and/or unrestricted research grants from Geistlich Biomaterials, Wolhusen, Switzerland; Osteology Foundation, Lucerne, Switzerland; Straumann, Freiburg, Germany; Dentsply Implants, Mannheim, Germany; and Camlog Biotechnologies, Wimsheim, Germany. The authors declare that they have no competing interests related to this systematic review.

\section{Authors' contributions}

HT has written the manuscript and calculated the statistics. FW has managed the literature search and collected the original literature. Both have made substantial contributions to study conception, interpretation of data as well as manuscript preparation. Both authors read and approved the final manuscript.

\section{Acknowledgements}

The authors appreciate the additional information provided during the process from Dr. Birgitta Bergendal, Övertandläkare, Odontologiska Institutionen, Jönköping, Sweden.

\section{Funding information}

This study is funded by the German Association of Dental Implantology (Deutsche Gesellschaft für Implantologie im Zahn-, Mund- und Kieferbereich e.V.)

\section{Author details}

'Department of Oral and Maxillofacial Surgery, Red Cross Hospital, Hansteinstr. 29, D-34121 Kassel, Germany. ${ }^{2}$ Department of Oral and Maxillofacial Surgery, Schleswig-Holstein University Hospital, Arnold-Heller-Straße 3, Haus 26, 2D-4105 Kiel, Germany.

Received: 25 July 2015 Accepted: 27 August 2015

Published online: 18 November 2015

\section{References}

1. Créton M, Cune M, Verhoeven W, Muradin M, Wismeijer D, Meijer G. Implant treatment in patients with severe hypodontia: a retrospective evaluation. J Oral Maxillofac Surg. 2010;68:530-8.

2. Bergendal B. Oligodontia ectodermal dysplasia—on signs, symptoms, genetics, and outcomes of dental treatment. Swed Dent J Suppl. 2010;205:13-78. 7-8.

3. Thilander B, Odman J, Lekholm U. Orthodontic aspects of the use of oral implants in adolescents: a 10-year follow-up study. Eur J Orthod. 2001;23:715-31.

4. Pigno MA, Blackman RB, Cronin Jr RJ, Cavazos E. Prosthodontic management of ectodermal dysplasia: a review of the literature. J Prosthet Dent. 1996;76:541-5.

5. Bergendal B. When should we extract deciduous teeth and place implants in young individuals with tooth agenesis? J Oral Rehabil. 2008;35 Suppl 1:55-63.

6. Kramer FJ, Baethge C, Tschernitschek H. Implants in children with ectodermal dysplasia: a case report and literature review. Clin Oral Implants Res. 2007;18:140-6.

7. Aydinbelge $M$, Gumus HO, Sekerci AE, Demetoğlu U, Etoz OA. Implants in children with hypohidrotic ectodermal dysplasia: an alternative approach to esthetic management: case report and review of the literature. Pediatr Dent. 2013;35:441-6.

8. Hobkirk JA, Nohl F, Bergendal B, Storhaug K, Richter MK. The management of ectodermal dysplasia and severe hypodontia. International conference statements. J Oral Rehabil. 2006:33:634-7.

9. Klineberg I, Cameron A, Hobkirk J, Bergendal B, Maniere MC, King N, et al. Rehabilitation of children with ectodermal dysplasia. Part 2: an international consensus meeting. Int J Oral Maxillofac Implants. 2013;28:1101-9.

10. Klineberg I, Cameron A, Whittle T, Hobkirk J, Bergendal B, Maniere MC, et al. Rehabilitation of children with ectodermal dysplasia. Part 1: an international Delphi study. Int J Oral Maxillofac Implants. 2013;28:1090-100.

11. Yap AK, Klineberg I. Dental implants in patients with ectodermal dysplasia and tooth agenesis: a critical review of the literature. Int J Prosthodont. 2009;22:268-76.

12. Moher D, Liberati A, Tetzlaff J, Altman DG, The PRISMA Group. Preferred Reporting Items for Systematic Reviews and Meta-Analyses: the PRISMA Statement. PLoS Med. 2009;6:e1000097. doi:10.1371/journal.pmed1000097.

13. Viswanathan M, Ansari MT, Berkman ND, Chang S, Hartling L, McPheeters LM, et al. Assessing the Risk of Bias of Individual Studies in Systematic Reviews of Health Care Interventions. Agency for Healthcare Research and Quality Methods Guide for Comparative Effectiveness Reviews. March 2012. AHRQ Publication No. 12-EHC047-EF. Available at: http://www.effectivehealthcare.ahrq.gov/ehc/products/322/998/ MethodsGuideforCERs_Viswanathan_IndividualStudies.pdf.

14. Buser D, Janner SF, Wittneben JG, Brägger U, Ramseier CA, Salvi GE. 10-year survival and success rates of 511 titanium implants with a sandblasted and acid-etched surface: a retrospective study in 303 partially edentulous patients. Clin Implant Dent Relat Res. 2012;14:839-51.

15. Grecchi F, Zingari F, Bianco R, Zollino I, Casadio C, Carinci F. Implant rehabilitation in grafted and native bone in patients affected by ectodermal dysplasia: evaluation of 78 implants inserted in 8 patients. Implant Dent. 2010;19:400-8.

16. Hvaring CL, Øgaard B, Stenvik A, Birkeland K. The prognosis of retained primary molars without successors: infraocclusion, root resorption and restorations in 111 patients. Eur J Orthod. 2014;36:26-30.

17. Kjaer I, Nielsen MH, Skovgaard LT. Can persistence of primary molars be predicted in subjects with multiple tooth agenesis? Eur J Orthod. 2008;30:249-53. 
18. Dellavia C, Catti F, Sforza C, Tommasi DG, Ferrario VF. Craniofacial growth in ectodermal dysplasia. Angle Orthod. 2010;80:733-9.

19. Dellavia C, Catti F, Sforza C, Grandi G, Ferrario VF. Non-invasive longitudinal assessment of facial growth in children and adolescents with hypohidrotic ectodermal dysplasia. Eur J Oral Sci. 2008;116:305-11.

20. Bergendal B, Ekman A, Nilsson P. Implant failure in young children with ectodermal dysplasia: a retrospective evaluation of use and outcome of dental implant treatment in children in Sweden. Int J Oral Maxillofac Implants. 2008;23:520-4.

21. Durstberger G, Celar A, Watzek G. Implant-surgical and prosthetic rehabilitation of patients with multiple dental aplasia: a clinical report. Int J Oral Maxillofac Implants. 1999;14:417-23.

22. Grecchi F, Pagliani L, Mancini GE, Zollino I, Carinci F. Implant treatment in grafted and native bone in patients affected by ectodermal dysplasia. J Craniofac Surg. 2010;21(6):1776-80.

23. Bauss O, Engelke W, Fenske C, Schilke R, Schwestka-Polly R. Autotransplantation of immature third molars into edentulous and atrophied jaw sections. Int J Oral Maxillofac Surg. 2004;33:488-96.

24. Bokelund M, Andreasen JO, Christensen SS, Kjaer I. Autotransplantation of maxillary second premolars to mandibular recipient sites where the primary second molars were impacted, predisposes for complications. Acta Odontol Scand. 2013;71:1464-8.

25. Krieger O, Matuliene G, Hüsler J, Salvi GE, Pjetursson B, Brägger U. Failures and complications in patients with birth defects restored with fixed dental prostheses and single crowns on teeth and/or implants. Clin Oral Implants Res. 2009;20:809-16.

26. Dueled E, Gotfredsen K, Trab Damsgaard M, Hede B. Professional and patient-based evaluation of oral rehabilitation in patients with tooth agenesis. Clin Oral Implants Res. 2009;20:729-36.

27. Finnema KJ, Raghoebar GM, Meijer HJ, Vissink A. Oral rehabilitation with dental implants in oligodontia patients. Int J Prosthodont. 2005;18:203-9

28. Goshima K, Lexner MO, Thomsen CE, Miura H, Gotfredsen K, Bakke M. Functional aspects of treatment with implant-supported single crowns: a quality control study in subjects with tooth agenesis. Clin Oral Implants Res. 2010;21:108-14.

29. Robertsson S, Mohlin B. The congenitally missing upper lateral incisor. A retrospective study of orthodontic space closure versus restorative treatment. Eur J Orthod. 2000;22:697-710.

30. Uribe F, Chau V, Padala S, Neace WP, Cutrera A, Nanda R. Alveolar ridge width and height changes after orthodontic space opening in patients congenitally missing maxillary lateral incisors. Eur J Orthod. 2013;35:87-92.

31. Uribe F, Padala S, Allareddy V, Nanda R. Cone-beam computed tomography evaluation of alveolar ridge width and height changes after orthodontic space opening in patients with congenitally missing maxillary lateral incisors. Am J Orthod Dentofacial Orthop. 2013;144:848-59.

32. Johnson EL, Roberts MW, Guckes AD, Bailey L, Phillips CL, Wright JT. Analysis of craniofacial development in children with hypohidrotic ectodermal dysplasia. Am J Med Genet. 2002;112:327-34.

33. Wittneben JG, Millen C, Brägger U. Clinical performance of screw- versus cement-retained fixed implant-supported reconstructions-a systematic review. Int J Oral Maxillofac Implants. 2014;29(Suppl):84-98.

34. Pjetursson BE, Tan WC, Zwahlen M, Lang NP. A systematic review of the success of sinus floor elevation and survival of implants inserted in combination with sinus floor elevation. J Clin Periodontol. 2008:35(Suppl):216-40

35. Bergendal B. Interpretive and report bias in publications on implants in patients with ectodermal dysplasia. Int J Prosthodont. 2011:24:505-6.

36. Jabbour Z, Emami E, de Grandmont P, Rompré PH, Feine JS. Is ora health-related quality of life stable following rehabilitation with mandibular two-implant overdentures? Clin Oral Implants Res. 2012;23:1205-9.

37. Hashem A, Kelly A, O'Connell B, O'Sullivan M. Impact of moderate and severe hypodontia and amelogenesis imperfecta on quality of life and self-esteem of adult patients. J Dent. 2013;41:689-94.

38. Heuberer S, Dvorak G, Zauza K, Watzek G. The use of onplants and implants in children with severe oligodontia: a retrospective evaluation. Clin Oral Implants Res. 2012;23:827-31.

39. Antonarakis GS, Prevezanos P, Gavric J, Christou P. Agenesis of maxillary lateral incisor and tooth replacement: cost-effectiveness of different treatment alternatives. Int J Prosthodont. 2014;27:257-63.
40. Ledermann PD, Hassell TM, Hefti AF. Osseointegrated dental implants as alternative therapy to bridge construction or orthodontics in young patients: seven years of clinical experience. Pediatr Dent. 1993;15:327-33.

41. Kearns G, Sharma A, Perrott D, Schmidt B, Kaban L, Vargervik K. Placement of endosseous implants in children and adolescents with hereditary ectodermal dysplasia. Oral Surg Oral Med Oral Pathol Oral Radiol Endod. 1999:88:5-10.

42. Guckes AD, Scurria MS, King TS, McCarthy GR, Brahim JS. Prospective clinical trial of dental implants in persons with ectodermal dysplasia. J Prosthet Dent. 2002;88:21-5.

43. Sweeney IP, Ferguson JW, Heggie AA, Lucas JO. Treatment outcomes for adolescent ectodermal dysplasia patients treated with dental implants. Int J Paediatr Dent. 2005;15:241-8.

44. Poggio CE, Salvato M, Salvato A. Multidisciplinary treatment of agenesis in the anterior and posterior areas: a long term retrospective analysis. Prog Orthod. 2005:6:262-9.

45. Zarone F, Sorrentino R, Vaccaro F, Russo S. Prosthetic treatment of maxillary lateral incisor agenesis with osseointegrated implants: a 24-39-month prospective clinical study. Clin Oral Implants Res. 2006;17:94-101.

46. Becelli R, Morello R, Renzi G, Dominici C. Treatment of oligodontia with endo-osseous fixtures: experience in eight consecutive patients at the end of dental growth. J Craniofac Surg. 2007:18:1327-30.

47. Degidi M, Nardi D, Piattelli A. Immediate versus one-stage restoration of small-diameter implants for a single missing maxillary lateral incisor: a 3-year randomized clinical trial. J Periodontol. 2009;80:1393-8.

48. Nissan J, Mardinger O, Strauss M, Peleg M, Sacco R, Chaushu G. Implantsupported restoration of congenitally missing teeth using cancellous bone block-allografts. Oral Surg Oral Med Oral Pathol Oral Radiol Endod. 2011:111:286-91.

49. Hosseini M, Worsaae N, Schiødt M, Gotfredsen K. A 3-year prospective study of implant-supported, single-tooth restorations of all-ceramic and metal-ceramic materials in patients with tooth agenesis. Clin Oral Implants Res. 2013;24:1078-87.

50. Zou D, Wu Y, Wang XD, Huang W, Zhang Z, Zhang Z. A retrospective 3- to 5-year study of the reconstruction of oral function using implant-supported prostheses in patients with hypohidrotic ectodermal dysplasia. J Oral Implantol. 2014;40:571-80.

51. Kristerson L, Lagerström L. Autotransplantation of teeth in cases with agenesis or traumatic loss of maxillary incisors. Eur J Orthod. 1991;13:486-92

52. Kugelberg R, Tegsjö U, Malmgren $\mathrm{O}$. Autotransplantation of 45 teeth to the upper incisor region in adolescents. Swed Dent $J$. 1994;18:165-72.

53. Marcusson KAM, Lilja-Karlander EK. Autotransplantation of premolars and molars in patients with tooth aplasia. J Dent. 1996;24:355-8

54. Josefsson E, Brattström V, Tegsjö U, Valerius-Olsson H. Treatment of lower second premolar agenesis by autotransplantation: four-year evaluation of eighty patients. Acta Odontol Scand. 1999;57:111-5.

55. Czochrowska EM, Stenvik A, Bjercke B, Zachrisson BU. Outcome of tooth transplantation: survival and success rates $17-41$ years posttreatment. Am J Orthod Dentofacial Orthop. 2002;121:110-9.

56. Jonsson T, Sigurdsson TJ. Autotransplantation of premolars to premolar sites. A long-term follow-up study of 40 consecutive patients. Am J Orthod Dentofacial Orthop. 2004;125:668-75.

57. Tanaka T, Deguchi T, Kageyama T, Kanomi R, Inoue M, Foong KW. Autotransplantation of 28 premolar donor teeth in 24 orthodontic patients. Angle Orthod. 2008;78:12-9.

58. Mensink G, Merkesteyn R. Autotransplantation of premolars. Brit Dent J. 2010;208:109-11.

59. Kvint S, Lindsten R, Magnusson A, Nilsson P, Bjerklin K. Autotransplantation of teeth in 215 patients. A follow-up study. Angle Orthod. 2010;80:446-51.

60. Bjerklin K, Bennett J. The long-term survival of lower second primary molars in subjects with agenesis of the premolars. Eur J Orthod. 2000:22:245-55.

61. Ith-Hansen K, Kjaer I. Persistence of deciduous molars in subjects with agenesis of the second premolars. Eur J Orthod. 2000;22:239-43.

62. Sletten DW, Smith BM, Southard KA, Casko JS, Southard TE. Retained deciduous mandibular molars in adults: a radiographic study of long-term changes. Am J Orthod Dentofacial Orthop. 2003;124:625-30. 
63. Bjerklin K, Al-Najjar M, Kårestedt H, Andrén A. Agenesis of mandibular second premolars with retained primary molars: a longitudinal radiographic study of 99 subjects from 12 years of age to adulthood. Eur J Orthod. 2008;30:254-61.

64. Hobkirk JA, Goodman JR, Reynolds IR. Component failure in removable partial dentures for patients with severe hypodontia. Int J Prosthodont 1989;2:327-30

65. Pröbster B, Henrich GM. 11-year follow-up study of resin-bonded fixed partial dentures. Int J Prosthodont. 1997;10:259-68.

66. Garnett MJ, Wassell RW, Jepson NJ, Nohl FS. Survival of resin-bonded bridgework provided for post-orthodontic hypodontia patients with missing maxillary lateral incisors. Br Dent J. 2006;21(201):527-34.

67. Spinas E, Aresu M, Canargiu F. Prosthetic rehabilitation interventions in adolescents with fixed bridges: a 5-year observational study. Eur J Paediatr Dent. 2013;14:59-62

68. Wong AT, McMillan AS, McGrath C. Oral health-related quality of life and severe hypodontia. J Oral Rehabil. 2006;33:869-73.

69. Stanford CM, Guckes A, Fete M, Srun S, Richter MK. Perceptions of outcomes of implant therapy in patients with ectodermal dysplasia syndromes. Int J Prosthodont. 2008;21:195-200.

70. Locker D, Jokovic A, Prakash P, Tompson B. Oral health-related quality of life of children with oligodontia. Int J Paediatr Dent. 2010;20:8-14

71. Laing E, Cunningham SJ, Jones S, Moles D, Gill D. Psychosocial impact of hypodontia in children. Am J Orthod Dentofacial Orthop. 2010;137:35-41.

72. Kohli R, Levy S, Kummet CM, Dawson DV, Stanford CM. Comparison of perceptions of oral health-related quality of life in adolescents affected with ectodermal dysplasia relative to caregivers. Spec Care Dentist. 2011:31:88-94

73. Meaney S, Anweigi L, Ziada H, Allen F. The impact of hypodontia: a qualitative study on the experiences of patients. Eur J Orthod. 2012;34:547-52.

74. Anweigi L, Finbarr Allen $\mathrm{P}$, Ziada $\mathrm{H}$. Impact of resin bonded bridgework on quality of life of patients with hypodontia. J Dent. 2013;41:683-8.

75. Anweigi L, Allen PF, Ziada H. The use of the Oral Health Impact Profile to measure the impact of mild, moderate and severe hypodontia on oral health-related quality of life in young adults. J Oral Rehabil. 2013;40:603-8.

\section{Submit your manuscript to a SpringerOpen ${ }^{\circ}$ journal and benefit from:}

- Convenient online submission

- Rigorous peer review

- Immediate publication on acceptance

- Open access: articles freely available online

- High visibility within the field

- Retaining the copyright to your article

Submit your next manuscript at $>$ springeropen.com 